\title{
Efficiency Gains through Social Influence in a Minimum Effort Game
}
J. Arifovic
H. Dawid
M. Nanumyan 


\title{
Efficiency Gains through Social Influence in a Minimum Effort Game
}

\author{
Jasmina Arifovic* $^{*}$ Herbert Dawid ${ }^{\dagger} \quad$ Mariam Nanumyan $^{\ddagger}$
}

January 2020

\begin{abstract}
In this paper we explore the role of social influence for the coordination of effort choice in a game with strategic complementarities. Players are repeatedly randomly partitioned in groups to play a minimum effort game and choose their effort based on their beliefs about the minimal effort among the other members of their group. Individual expectations about this minimal effort is influenced by own experience as well as by communication of beliefs within a social network. We show that increasing the importance of social influence in the expectation formation process has positive effects on the emerging (long run) effort level, thereby improving the efficiency of the outcome. Furthermore, a more centralized social network leads to higher average efficiency, but also to increased variance of outcomes. Finally, communication of actual minimum effort cannot replace the communication of beliefs as a device fostering the emergence of high long run effort.
\end{abstract}

JEL Classification: C63, C73, D83

${ }^{*}$ Department of Economics, Simon Fraser University, Burnaby, Canada. Email: jasmina_arifovic@sfu.ca

${ }^{\dagger}$ Department of Business Administration and Economics and Center for Mathematical Economics, Bielefeld University, Germany. Email: hdawid@uni-bielefeld.de

${ }^{\ddagger}$ Department of Business Administration and Economics, Bielefeld University, Germany and Center for Operations Research and Econometrics, Université catholique de Louvain, Belgium. Email: mariam@nanumyan.com 
Keywords: Minimum Effort Game, Expectation Formation, Social Influence, Belief Communication

\section{Introduction}

Group production plays a central role in many economic contexts. In the presence of strategic complementarities between the actions of group members, the problem of determining individual effort by each member in the group typically gives rise to multiple (Pareto ranked) equilibria and hence to a severe coordination problem. In particular, the optimal effort choice of an agent is crucially determined by her beliefs about the effort the other group members invest into the project. Taking this into account, the way expectations are formed and adjusted over time is a key driver for determining the outcome of (repeated) group production problems. In particular, whether an efficient outcome can be reached in the long run strongly depends on the evolution of the individual expectations of the players.

The agenda of this paper is to study the role of one important aspect of expectation formation, namely social influence, in the dynamics of expectations and actions in a population of agents which repeatedly undertake some joint production task with strategic complementarities in changing groups. As an illustrative example of a situation we have in mind we envision a university which repeatedly encounters calls for interdisciplinary project proposals and for each of these calls identifies a group of faculty members from different departments who, based on their background, are suitable to contribute to the proposal. Each group member then decides how much effort to invest in developing her part of the proposal. After submission the proposal is evaluated and the group receives an outcome (e.g. amount of funding, invitation to resubmit, rejection). We assume that referees tend to focus in their decision to a large extend on potential weaknesses they see, such that the outcome is determined by the lowest effort shown 
by any group member. Faculty members over time repeatedly participate in different such group proposals and over time build expectations about the minimal effort shown among the other members of their group. We assume that these expectations are not only based on their own experience, i.e. the outcome of the projects they have been previously involved in, but also on communication with their friends, close colleagues and co-authors in the profession about their experience with similar project proposals. In particular, we assume that agents communicate their own beliefs about the level of minimal effort shown in such an interdisciplinary group to their social contacts. We denote such kind of communication as the social influence channel of the expectation formation process. Whereas the groups jointly producing the project proposals differ from call to call, we assume that the set of social contacts of an agent stays constant over time. The main questions we address within such a setting are, whether the quality of the project proposals in the long run is higher if there is communication of the agents' own beliefs in the social network, and, how the outcome is affected by the topology of the social network.

Our research agenda builds on a large body of empirical and theoretical work studying the role of social influence for opinion dynamics and expectation formation. Starting with the seminal contribution of DeGroot (1974) there is by now a rich body of literature highlighting how communication in social networks affects the dynamics of opinion formation, in particular the emergence of consensus in a population (see e.g. the survey Acemoglu and Ozdaglar (2011)), and under which circumstances there is 'wisdom of the crowd' in the sense that communication in a network allows the agents to learn the true state of the world (e.g. Golub and Jackson (2010)). As shown e.g. in Golub and Jackson (2010) or Acemoglu et al. (2010) also the topology of the social network matters with respect to these issues. Recent contributions have also stressed that the impact of social influence on 
economic expectations can have important implications for actual dynamics in different economic contexts (e.g. Arifovic et al. (2010); Burnside et al. (2016); Rotemberg (2017)). However, so far a systematic analysis of the implications of social influence for the selection of the outcome emerging in a population faced with a group coordination problem is missing. The main contribution of this paper is to fill this gap.

We address our main research questions by considering a dynamic model of a population of agents, which every period is randomly partitioned into groups of given size. In each group agents interact by playing a minimum effort game with a finite set of effort choices. We use the minimum effort game as the most widely used model in the theoretical (starting with Bryant (1983)) and experimental (starting with Van Huyck et al. (1990)) literature capturing a group coordination problem with strategic complementarities and multiple Pareto ranked equilibria. Each agent in each period plays the best response to her current expectations. These expectations have the form of a belief distribution over the set of possible effort choices, expressing the probability that a given effort level is the minimum of the effort choices of the other members of the group. In each group only the outcome, i.e. the minimal effort level in the group, is observable for its members. At the end of each period agents update their expectations in two steps. First, in line with standard adaptive expectations models ${ }^{1}$, agents build intermediate beliefs as a weighted average of their previous beliefs and the observed outcome in the current period. In a second step, agents might communicate their intermediate beliefs with all their contacts in a social network, which is exogeneously given and constant over time. Following a standard approach in the literature on social influence dynamics introduced in DeGroot (1974) agents put identical weight on the intermediate beliefs of all their contacts

\footnotetext{
${ }^{1}$ See e.g. Huyck and Stahl (2018a) for a recent contribution in the framework of minimum effort games.
} 
and the updated belief is a weighted average of their own intermediate belief and average intermediate beliefs of their contacts. ${ }^{2}$ The weight agents put on the average beliefs of their social contacts determines the importance of social influence in the population. A main goal of our analysis is to understand how an increase in this social influence parameter affects the evolution of expectations and the distribution of effort levels chosen in the long run. We explore this question by combining analytical findings with insights from statistical analysis of data obtained through extensive simulations of the model under different assumptions about the size of the social influence parameter and the social network topology.

Since the focus of our analysis is on the role of social influence, we consider a model setup which, apart from the considered communication of beliefs, is as simple as possible and directly corresponding to the baseline stag hunt game, for which the experimental results of Van Huyck et al. (1990) show convergence to the least efficient equilibrium under large group sizes. Thereby, we do not incorporate into our model several mechanisms which have been shown in the literature to improve the efficiency of the emerging long run outcome of the game. In particular, our assumption that each agent considers only the own payoff when determining a best response

\footnotetext{
${ }^{2}$ This model of belief diffusion is sometimes referred to as 'naive learning' since individuals do not take into account that due to the structure of the social network there might be differences in the correlations between the intermediate beliefs of different pairs of their contacts, which should be reflected in the weights put on their intermediate beliefs. Alternatively a Bayesian Approach could be employed in which agents take into account the network structure in a fully rational way. As pointed out in DeMarzo et al. (2003) however a very high degree of rationality on the agents' side has to be assumed for them to infer the correct weights to be put on all their contacts. Indeed, as is shown in Grimm and Mengel (2019), the naive approach put forward in DeGroot (1974) is better able to explain the data from experiments studying the effect of social influence than a Bayesian model. Hence, we stick in our analysis to the assumption that identical weights are put on the communications from all social contacts.
} 
and then always chooses that best response does neither consider social preferences (see e.g. Chen and Chen (2011)) nor stochastic choice by agents, e.g. based on a the well studied logit model (e.g. Anderson et al. (2001); Huyck and Stahl (2018b)). Furthermore, we assume that an exogenous process stochastically determines the interaction group every period, while it has been shown in Riedel et al. (2016) that endogenous partner choice can improve the efficiency in coordination games. Agents in our setup with social influence communicate at the end of each period with their social contacts about their own beliefs, however there is no pre-play communication within each interaction group, which might improve the effort level chosen in the game (see e.g. Blume and Ortmann (2007); Kriss et al. (2016)). Also, in our setting agents do not condition their effort on the fraction of members of their interaction group with whom they have direct ties in the social network. This assumption is due to the fact that we consider large interaction groups where this fraction is typically small. ${ }^{3}$ Overall, by abstracting from all these effects and considering a very basic environment we are able to isolate the effect of social influence for the agent's effort choice.

The first main insight from our analysis is that in the absence of social influence, i.e. if the value of the social influence parameter is zero, the effort level in the population converges to the lowest effort level chosen in the entire population in the initial period. In accordance with the experimental evidence of Van Huyck et al. (1990), this implies that for large groups the long run effort level coincides with high probability with the lowest possible value and therefore the least efficient equilibrium is reached. Intuitively, in the absence of communication with their social contacts, agents observe only the outcome of the game in their own group, which corresponds to the lowest effort shown by any group member. Hence, the agent with the most pes-

\footnotetext{
${ }^{3}$ For small group sizes, in particular interaction groups of size two, experimental findings, e.g. by Chen and Chen (2011) show that effort in minimum effort games tends to be higher if a player is matched with another player belonging to the same identity group.
} 
simistic belief in the whole population never receives information that could move her best response upwards. Since every period all agents matched with this most pessimistic agent observe the group outcome corresponding to this agent's (low) effort, their beliefs become more pessimistic. Due to this mechanism all agents adjust their efforts downwards over time until it matches the lowest effort level in the population. The picture changes radically if there is social influence in the population. First, due to learning the expectations of her social contacts, an agent's beliefs can become more optimistic even if the observed outcome in the own group was low in the previous period. Second, due to the communication between agents, the beliefs in the population become homogeneous much faster. This induces a fast coordination on an equilibrium and avoids the 'downward drift' of beliefs over time, which occurs in heterogeneous populations due to the fact that in every group the observed outcome corresponds to the lowest effort in the group. We show that an increase of the social influence parameter induces statistically significantly higher long run effort in the population. Furthermore, our analysis establishes that the topology of the social network matters. In particular, the expected long run effort of agents in the population is significantly larger in a centralized star network compared to a random network. However, this increase of average efficiency comes at the cost of less predictability of the outcome in the sense that the variance of the long run outcome across simulation runs is much larger under a centralized network. Intuitively, in a centralized network the population is strongly influenced by the initial beliefs of the agent in the center of the network. This fosters fast coordination, but at the same time introduces a strong dependency of the long run outcome from the (stochastic) initial beliefs of a single agent. We show that our findings are not only robust with respect to variations of the model parameters, but also with respect to a change in the model setup, where agents do not only exchange their beliefs with their 
social contacts but also are able to observe the outcomes of all the group interactions in which any of their social contacts are involved. In particular, this implies that exchange of information about the outcomes of the group interaction in the social network cannot substitute the communication of beliefs in fostering coordination on more efficient equilibria.

The paper is organized as follows. In Section 2 we describe our model setting. In Section 3 we derive several analytical findings about the long run outcomes for special cases of our setting, including the scenario without social influence. In Section 4 we analyze the general case with social influence and in Section 5 we consider a model extension, where not only beliefs but also information about the actual outcomes of the group interactions are communicated in the social network. Concluding remarks are given in Section 6. Appendix A provides the proofs of the propositions in Section 3. In Appendix B we provide the statistical test results discussed in the paper and in Appendix $\mathrm{C}$ the robustness of our results with respect to parameter variations is demonstrated.

\section{Model}

\subsection{Setting}

There is a set of agents $\mathrm{N}$, with $|N|=n$, connected within a social network $s$. The set of connections of agent $i$ in the social network is fixed over time. We denote the set of social contacts of agent $i$ by $m_{i}(s)=\{j \mid i j \in s\}$ and by $\eta_{i}(s)=\left|m_{i}(s)\right|$ the number of social contacts of agent $i$.

Every period the set of agents is randomly partitioned into $n / k$ groups of size $k$, where each partition has equal probability. ${ }^{4}$ Thus, each agent faces an equal probability of being a member of each group. We denote by

\footnotetext{
${ }^{4}$ Assuming that $k$ is a divisor of $n$, the number of possible ways to form such partitions is given by $\left(\prod_{j=0}^{\frac{n}{k}-1}\left(\begin{array}{c}n-j k \\ k\end{array}\right)\right) /(n / k)$ !.
} 
$g(i, t) \subset N$ the set of members of the group to which agent $i$ belongs at $t$ (this implies $i \in g(i, t)$ for all $t$ ).

At each period each group plays the following minimum effort game (see Van Huyck et al. (1990)). Each agent $i$ chooses an effort $e_{i, t}$ from a given set of strategies $\mathcal{X}=\{1, \ldots, \bar{e}\}$. The payoff of the agent from the game is determined by her own effort and the minimal effort chosen by the members of her group in period $t$, denoted by $\underline{e}_{i, t}$. The payoff of agent $i$ is given by:

$$
\pi\left(e_{i, t}, e_{-i, t}\right)=\alpha \underline{e}_{i, t}-\beta e_{i, t},
$$

with $\alpha>\beta>0, e_{-i, t}=\left(e_{j, t}\right)_{j \in g(i, t) \backslash\{i\}} \quad$ and $\underline{e}_{i, t}=\min _{j \in g(i, t)} e_{j, t}$ is the minimum effort in the group of agent $i$.

\subsection{Beliefs}

Each agent at each $t$ has a belief about the distribution of minimal effort $\underline{e}$ in her (randomly generated) group: $b_{i, t} \in \Delta(\mathcal{X}):=\left\langle b \in \mathbb{R}_{+}^{\bar{e}}: \sum_{e=1}^{\bar{e}} b(e)=1\right\rangle$. We denote by $b_{i, t}(e)$ the probability that the minimal effort of the other players in the group is $e$. A belief vector which puts probability one on some effort level $\tilde{e}$ will be referred to as a point belief and formally written as $b=\mathbb{1}_{\tilde{e}}$. The population profile of beliefs at $t$ is denoted by $B_{t}=\left(b_{i, t}\right)_{i \in N}$.

An agent has no information about effort level chosen by individual agents and hence their beliefs about the distribution of minimal effort by the other members of their current group does not depend on the identities of these members. The expected payoff of an agent with effort $e \in \mathcal{X}$ and belief $b \in \Delta(\mathcal{X})$ is given by:

$$
\pi^{e}(e, b)=\sum_{\underline{e} \in \mathcal{X}} \pi(e, \underline{e}) b(\underline{e}) .
$$

Each agent $i$ chooses her effort by maximizing this expected payoff using her current belief distribution $b_{i, t}$. Hence, an agent with belief $b$ chooses an 
action $a^{*}(b)$ such that:

$$
\left.a^{*}(b)=\max \left\{e \in \mathcal{X} \mid \pi^{e}(e, b) \geq \pi^{e}(\tilde{e}, b)\right) \forall \tilde{e} \in \mathcal{X} .\right\}
$$

We denote the action of agent $i$ at $t$ by $a_{i, t}=a^{*}\left(b_{i, t}\right)$. Note that the above formulation of $a^{*}(b)$ implies that in case an agent is indifferent between different levels of efforts she always chooses the largest of these levels.

After the game has been played, all agents update their belief distributions. They utilize both the acquired information about the minimum effort in their group, and the information about the beliefs of their neighbors in the network. Precisely, each agent $i$ forms an intermediate belief $\tilde{b}_{i, t}$ as a weighted average of the previous belief and her current observation $\underline{e}_{i, t}$ :

$$
\tilde{b}_{i, t+1}=(1-\xi) b_{i, t}+\xi \mathbb{1}_{\underline{e}_{i, t}},
$$

where $0 \leq \xi \leq 1$ denotes the speed of individual updating. The fact that only the minimum effort, rather than all individual effort choices of $g(i, t)$, is used to update the beliefs is based on the assumption that the individual efforts of the group members are not observable. The only information available is the outcome of the game, which is determined by the minimum effort of all group members. ${ }^{5}$

Furthermore, individuals change beliefs due to social influence. In particular, they learn about intermediate beliefs $\tilde{b}_{j, t+1}(e)$ of their social contacts and use them to form their own final beliefs. Following standard formulations in the literature on opinion formation (e.g. DeGroot (1974)) we assume that the updated belief is a linear combination of the agent's intermediate belief and that of her social contacts. Formally, we have:

$$
b_{i, t+1}=\tilde{b}_{i, t+1}+\chi \frac{1}{\eta_{i}(s)} \sum_{j \in m_{i}(s)}\left(\tilde{b}_{j, t+1}-\tilde{b}_{i, t+1}\right) .
$$

\footnotetext{
${ }^{5}$ This assumption seems realistic in many examples of projects carried out by groups, where it is impossible to identify the individual contributions to the success of the project.
} 
The parameter $\chi$ in (2) represents the level of confidence or trust in the beliefs of social contacts. It determines the importance of social influence and is a key parameter in our analysis. This updated belief distribution is then the basis for the agent's effort choice in period $t+1$.

\section{Analytical Results}

The evolution of the profile of belief vectors $B_{t}$ constitutes a Markov pro$\operatorname{cess}^{6}$ on the state space $\Delta(\mathcal{X})^{n}$. A general analytical characterization of the transient dynamics or the long run distribution of this process seems infeasible and therefore in Section 4 we will use simulations to gain insights in this respect. However, it is possible to derive general characterizations of absorbing sets of the process, and for the special case where $\chi=0$ also a description of the long-run outcome of beliefs and induced effort can be derived.

It is well known that for each effort level $e \in \mathcal{X}$ there exists a symmetric Nash equilibrium of the underlying minimal effort game, in which all players choose the effort $e$. These Nash equilibria are Pareto ranked and due to $\alpha>\beta$ the outcome is more efficient the higher the equilibrium effort. In our setting each such symmetric Nash equilibrium corresponds to a uniform population profile with $b_{i, t}=\mathbb{1}_{e}$ for all $i \in N$. Since $a^{*}\left(\mathbb{1}_{e}\right)=e$ and therefore $\underline{e}_{i}=e$ for all agents $i$ under such a belief profile, it follows directly that any such uniform profile is an absorbing state of the process $B_{t}$. However, as we will show below, the process does not necessarily reach a state with uniform point beliefs, or even with uniform induced actions in the long run, at least as long as the social influence parameter $\chi$ is positive.

\footnotetext{
${ }^{6}$ For each partition of the agents into groups of size $k$ the belief of each agent $i$ is given by (1) and (2) and therefore deterministic. Hence, the distribution of $B_{t+1}$ given $B_{t}$ is determined by the probabilities of all possible group partitions and the beliefs of all agents in $t+1$ given a certain partition.
} 
The following proposition shows that if all agents in the population in some period choose identical effort, they will all continue to choose this effort in all future periods as well.

Proposition 1. If at some $t \geq 0$ there exists an effort level $e \in \mathcal{X}$ such that $a^{*}\left(b_{i, t}\right)=e \forall i \in N$ then

(i) $a_{i, \tau}=e \forall i \in N$ for all $\tau \geq t$,

(ii) $b_{i, \tau} \rightarrow \mathbb{1}_{e}$ for $\tau \rightarrow \infty$ for all $i \in N$.

The intuition for this result is quite straight-forward. If there is no communication of beliefs, then in a period $t$, in which all agents observe a minimal effort $e$, the weight of this effort level in the updated belief distribution of every agent becomes larger. Due to the strategic complementarity this increases for all agents their incentive to choose that effort level in period $t+1$ and, since choosing $e$ has already been their optimal choice in $t$, they all choose effort $e$ also in period $t+1$.

Proposition 1 shows that any set of beliefs corresponding to some uniform choice of effort among agents is absorbing. This raises the question whether it is guaranteed that the belief process ends up in one of these absorbing states with uniform effort choice. In the absence of social influence the answer to this question is affirmative. In the following proposition we show that if $\chi=0$ the population always converges to a state in which all agents have identical point beliefs, i.e. they all expect with probability one that the minimum effort is some $\tilde{e} \in \mathcal{X}$ and choose their own effort level equal to $\tilde{e}$. Moreover, the long run effort is determined by the minimal effort chosen among all agents at $t=1$.

Proposition 2. Assume that $\chi=0$ and denote by $\underline{e}_{1}=\min _{i \in N}\left[a^{*}\left(b_{i, 1}\right)\right]$. Then,

(i) actions of an agent never increase over time: $a_{i, t+1} \leq a_{i, t}$ for all $i \in N, t \geq 1$. 
(ii) for every $\epsilon>0$ there exists $T>0$ such that $\mathbb{I P}\left[a_{i, \tau}=\underline{e}_{1} \forall i=1, . ., N\right.$ for all $\tau \geq t]>1-\epsilon$,

(iii) $b_{i, t} \rightarrow \mathbb{1}_{\underline{e}_{1}}$ (in probability) for $t \rightarrow \infty$ for all $i \in N$.

Proposition 2 shows that in the absence of social influence the population in the long run always coordinates on some effort level, which means that the population profile of beliefs always reaches a state corresponding to a Nash equilibrium of the game. However, the proposition also gives a clear indication that the dynamic adjustment of individual's beliefs induces a downward trend in the chosen effort and in the long run all agents adopt the smallest among all effort levels chosen in the initial period. The reason for this downward trend is that, due to the structure of the minimum effort game, no agent ever observes an outcome which is above its own effort level. In the absence of social influence this implies that when an agent updates her beliefs she always increases the weight of an effort level which is below or equal her best response in the current period. Hence, the best response of an agent can never increase over time. This induces contagion-like dynamics of agents switching to beliefs that induce the minimal effort in the population, which constantly remains at $\underline{e}_{1}$. Hence, in the long-run all agents in the population choose this minimal effort.

For a sufficiently large value of the social influence parameter $\chi$ the claim that beliefs and actions become uniform in the long are in general no longer true. Actions might stay heterogeneous forever and, therefore, also effort levels above the initial minimal effort are chosen by some agents in the long run. Defining $\underline{\chi}:=\frac{(n-2) \beta}{(n-k) \alpha}$ we obtain the following proposition.

Proposition 3. Assume that $(n-k) \alpha>(n-2) \beta$ and $\chi \geq \underline{\chi}>0$. Then for any initial belief profile $B_{1}$ with $\left|\left\{i \in N: a^{*}\left(b_{i, 1}\right)=\underline{e}_{1}\right\}\right|=1$ there exists a network $s$ such that $\max _{i \in N}\left[a_{i, t}\right]>\min _{i \in N}\left[a_{i, t}\right]$ for all $t$ with probability one. 
To interpret the proposition it should first be noted that the condition $(n-k) \alpha>(n-2) \beta$ implies that $\underline{\chi}<1$, such that the interval $(\underline{\chi}, 1]$ of values of the social influence parameter $\chi$ leading to heterogeneous long-run beliefs and actions is not empty. Given that we always have $\alpha>\beta$ the condition is quite weak as long as we assume that the size of the interaction group $(k)$ is small compared to the population size $(n)$. Whereas Proposition 3 is formulated for initial beliefs inducing that the minimal effort in the first period is chosen by a single agent, analogous results can be obtained for scenarios with a larger number of agents choosing the lowest initial effort $\underline{e}_{1}$ with an adjusted value of the threshold $\underline{\chi}$.

The intuition for the potential long-run heterogeneity of beliefs and actions in the presence of social influence is that in a situation, in which the beliefs of the social contacts of an agent are more optimistic than the observed outcome of the group interaction of that agent, the social influence, i.e. the direct communication of beliefs, might prevent the downward adjustment of that agent's beliefs. Hence the contagion of low effort choices, which drives the dynamics in the absence of social influence, might be stopped. If the social network is such that agents, which initially choose low effort do no have social ties to more optimistic individuals, which is the type of network on which the proof Proposition 3 is based, then neither do these agents increase their actions over time, nor do the more optimistic individuals, who are linked through the social network, adjust their beliefs so strongly downwards to choose the minimal population effort. Whenever these optimistic agents are in the same interaction group with the pessimistic agent, the negative impact of the observed low outcome in their group on their beliefs is outweighed by the social influence from their optimistic social contacts. Hence, in such a scenario long-run heterogeneity of beliefs and actions prevails.

Our analytical findings provide little guidance on the shape of the dy- 
namics and long run distribution of beliefs and actions for $\chi>0$. In the following section we employ numerical simulations to explore how the evolution of beliefs and efforts are affected by social influence in different settings. In particular, we examine how the degree of confidence in the beliefs of others, and the topology of the social network influence the distribution of minimum efforts and associated payoffs in the game.

\section{Effect of Social Influence}

The following analysis relies on a baseline parametrization of the model given by $n=30, k=5, \alpha=2, \beta=1, \bar{e}=15, \xi=0.1$ and $\chi=0.3$. The game parameters $\alpha$ and $\beta$ are chosen in line with Van Huyck et al. (1990). A relatively low speed of updating $\xi$ is chosen to prevent overly naive behavior of agents driven entirely by their previous period observation. Variations of the confidence parameter $\chi$ will be discussed extensively in the next section. The chosen values of $n, k$ and $\bar{e}$ turn out not to be crucial for the qualitative results we will discuss. Robustness checks showing that our results still hold for alternative specifications of the parameters are provided in Appendix C. Furthermore, we assume that all agents initially have heterogeneous point beliefs of the form $b_{i, 1}=\mathbb{1}_{\tilde{e}}$ for $\tilde{e}$ uniformly chosen from $\mathcal{X}$. Our comparison of model outcomes under different parameter and network constellations is based on batches of $Q=20$ simulation runs carried out for each constellation. In order to avoid spurious effects induced by different set of initial beliefs across the sets of batch runs, we generate a set of $Q$ initial beliefs (one for each batch run). We use these same initial beliefs in each set of batch runs carried out under the different considered parameter constellations. Concerning the social network, our benchmark is to consider a random network with linking probability $p=0.2$ between each pair of agents. Similarly to our approach taken with respect to initial beliefs we generate a set of $Q$ random networks and use this same set in every batch 


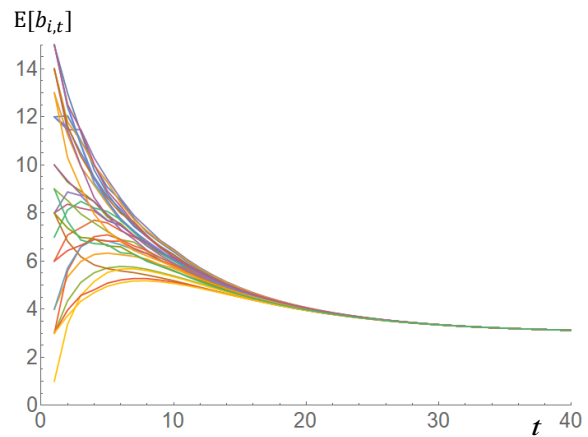

(a)

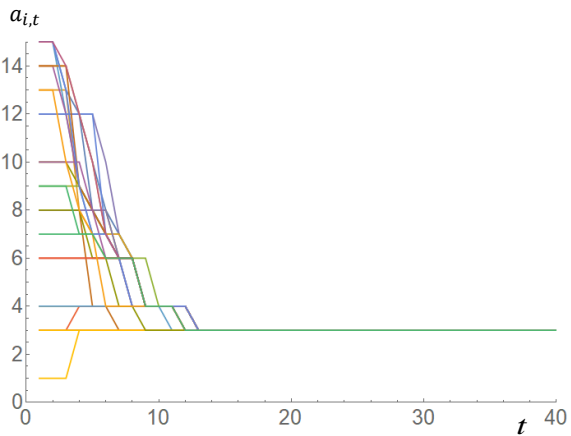

(b)

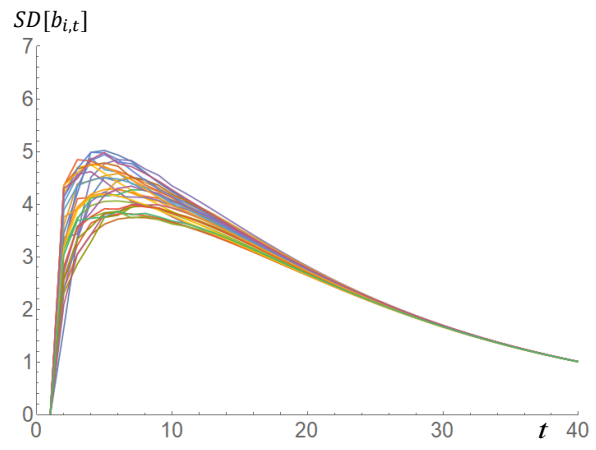

(c)

Figure 1: Dynamics of the expected values of the belief distributions (a), the chosen actions (b) and the standard deviation of the belief distributions (c) for all agents in a single run in the baseline scenario.

of run across different parameter constellations.

\subsection{Model Dynamics in the Baseline Scenario}

To gain some initial understanding of the mechanisms at work we first illustrate the dynamics of a single simulation run in the case of a random social network.

Figure 1 shows the dynamics of the action as well as the expected value and the standard deviation of the belief of each agent in the population. 
The figure illustrates that whereas agents start with heterogeneous point beliefs, initially the belief distributions of individual agents quickly become more distributed (i.e. the standard deviation of individual belief distributions increases, see panel (c)) and at the same time the expected values of individual beliefs approach each other (panel (a)). This is due to the interplay of observing the actual minimum effort in an agent's own group, which might differ from the agent's expectation, and the communication of beliefs from the agent's social contacts. Actions stay strongly heterogeneous for approximately 10 periods and then quickly converge to a common effort level of $e=3$ in this run. Once actions have converged to a uniform profile they stay constant over time (as shown in Proposition 1). Panel (b) of Figure 1 illustrates this result. The standard deviation of individual beliefs goes to zero (see panel(c)) and the expectations of the individual beliefs become uniform across agents slowly converging to the actual effort level observed in all groups (see panel (a)). In other words, the belief profile converges towards a profile of homogeneous point beliefs.

Agents with low point beliefs at $t=1$, due to social influence, quickly become more optimistic about the minimum effort in their group (see panel (a)). For some of them this leads to an increase of the chosen action over time (see panel (b)). In light of Proposition 2(i), which shows that in the absence of social influence individual effort levels can never increase over time, it is clear that this effect is driven by the communication of beliefs between agents. The intuition is similar to that already discussed at the end of Section 3. In a setting where outcomes are determined by the lowest effort in the group, like the minimum effort game, direct communication is important because it allows agents to realize that other agents in the population have expectations that are much more optimistic than observable outcomes would suggest. Hence, social influence might induce an upward adjustment of individual beliefs which is sufficiently strong to give rise to an 


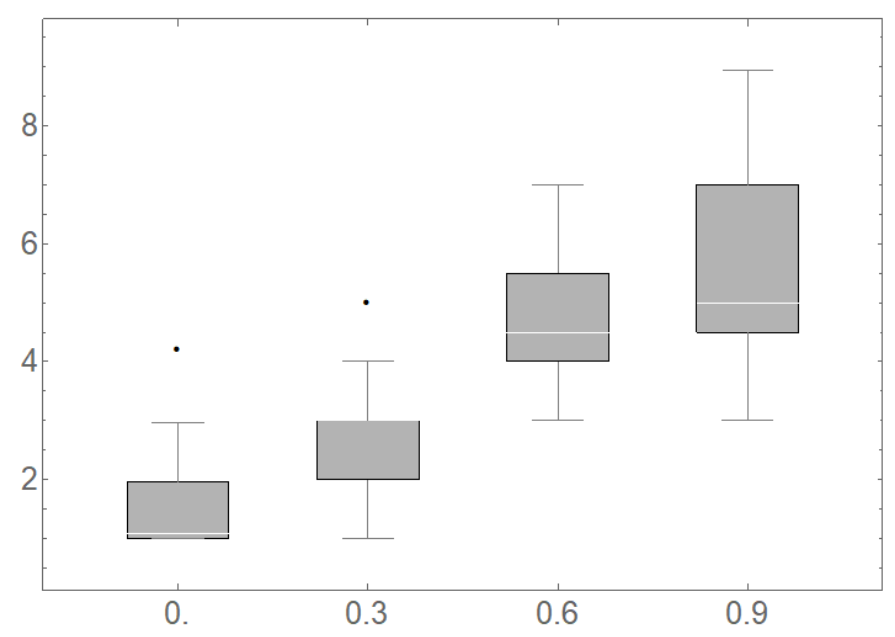

Figure 2: Distribution of average efforts at $t=40$ for $\chi=0,0.3,0.6,0.9$.

increase of the agent's (optimal) action choice.

\subsection{Effects of Social Influence}

The discussion of the single run in our baseline scenario highlights the strong importance of social influence for the dynamics of effort level choices in the population. To examine the role of social influence in more detail we now systematically analyze how the agents' level of confidence in the beliefs of their social contacts affects the emerging level of effort in the population. More precisely, we vary the confidence parameter $\chi$ between $\chi=0$ and $\chi=0.9$ and for each of the considered values carry out a batch of $Q=$ 20 simulation runs of $T=40$ periods. Figure 2 shows boxplots of the distributions of the population average of actions across the batch runs. It should be noted that in all runs at $t=40$ actions are already uniform such that the population average coincides with the action of every single agent in the population. It can be clearly seen that a higher level of social influence, expressed by a larger value of $\chi$, significantly increases the distribution of 


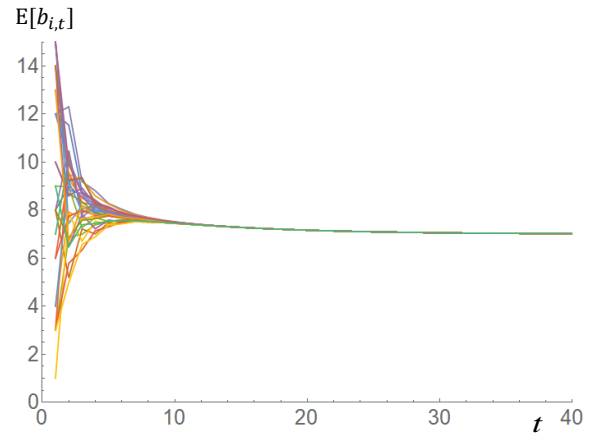

(a)

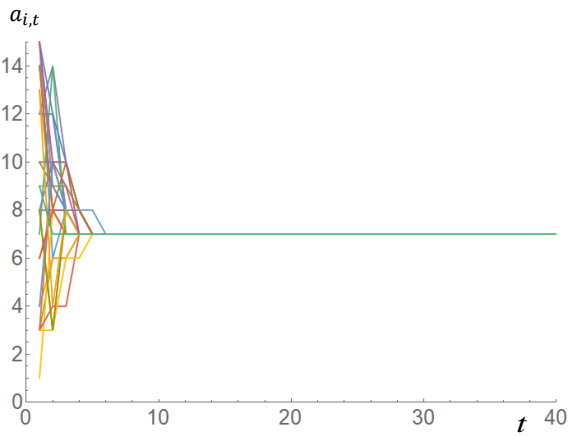

(b)

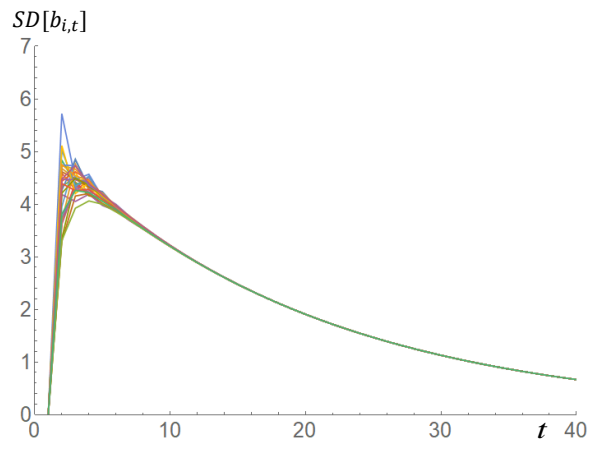

(c)

Figure 3: Dynamics of expected values of minimal effort (a), chosen actions (b) and standard deviation of the belief distributions (c) for all agents in a single run under strong social influence $(\chi=0.9)$.

long run efforts in the population. ${ }^{7}$

Similarly to Figure 1 described earlier, Figure 3 shows the dynamic of the action (panel (b)), expected value and the standard deviation of the belief of each agent in the population (panels (a) and (c)) where the level of confidence in social contacts' beliefs $\chi$ is equal to 0.9 compared to $\chi=$ 0.3 in the baseline scenario. In order to see the effect of increased social

\footnotetext{
${ }^{7}$ In Appendix B we provide the results of the Wilcoxon Signed Rank Tests to show that the distributions of effort are indeed significantly different for different values of $\chi$.
} 
influence one can first notice that the beliefs of the agents converge much faster with higher confidence level $\chi$. Particularly, the agents with low initial beliefs become more optimistic quicker and the beliefs across all agents converge faster. Based on this, the beliefs stay more optimistic in the long run compared to the benchmark in Figure 1 where the population variance of the beliefs decreases much slower. Panel (c) of Figure 3 shows that the beliefs of individual agents quickly become more dispersed reaching the peak of the standard deviations earlier compared to the benchmark, but this dispersion then is reduced more quickly than in the case of a low value of $\chi$.

The positive effect of the social influence on the pace of convergence and effort choice proves to be robust. Figure 4 shows for different values of $\chi$ the dynamics of distributions of average beliefs about the minimal effort across the agents in the network (panel (a)), average standard deviation of beliefs across the agents (panel (b)) and the standard deviation of the beliefs in the network (panel (c)). The figure illustrates confidence bands (across batch runs) of these values for baseline scenario (green bands), high level of social influence (blue bands) and the absence of social influence (grey bands). Similar to the single runs discussed above, the confidence bands for the (population) standard deviation of expectations about the minimum effort decreases much faster with higher level of social influence (see panel (c)). The distribution of average standard deviation of beliefs in the network reaches its maximum earlier and decreases much faster with higher value of $\chi$ (panel (b)). Finally, the beliefs converge to a significantly higher value of long run efforts when the level of trust in beliefs of the social contacts is high (panel (a)). Considering the initial periods in the dynamics of population mean of the expectations in Figure 4(a), it can be clearly seen that the average speed (across batch runs) of its decrease is almost identical for the different values of $\chi$. The crucial difference between the three considered scenarios is that for large values of $\chi$ the point in time, 


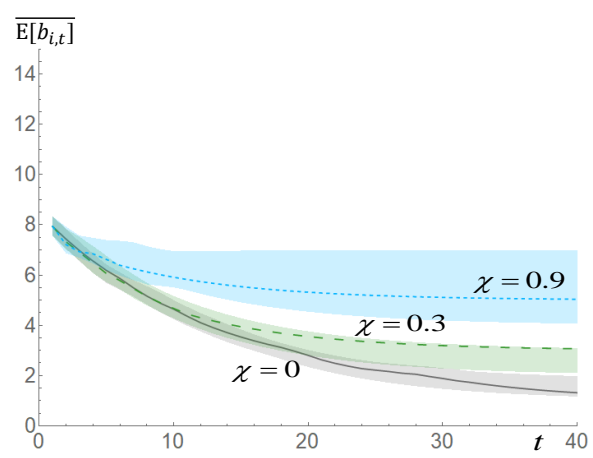

(a)

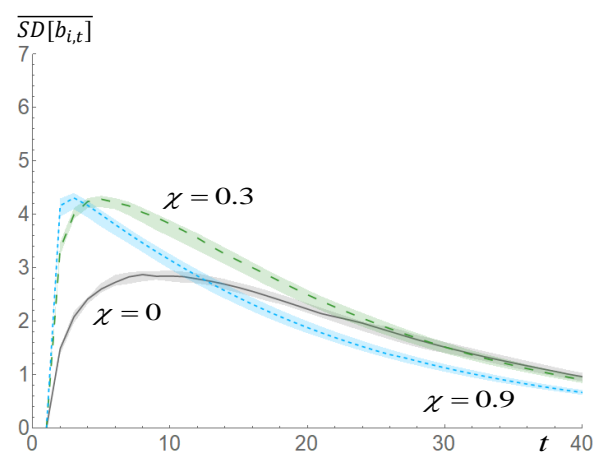

(b)

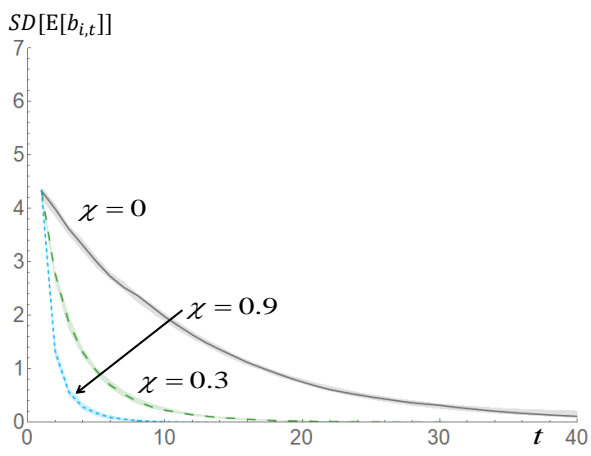

(c)

Figure 4: Dynamics of distribution of the population mean of expectation (a) and standard deviation of the individual belief distributions in the baseline scenario. Panel (c) shows the standard deviation of expected minimal effort across agents. The confidence bands in all panels illustrate the dynamics of the mean across the set of batch runs and one standard deviation from it for $\chi=0$ (grey), 0.3 (green) and 0.9 (blue).

where the population variance is close to zero, is much earlier and the mean expectation does hardly decrease further once the population has become almost uniform. This observation reinforces our intuition that a stronger effect of social influence improves the long-run effort level mainly by fostering faster convergence of population beliefs, thereby avoiding a long lasting drift 
towards a minimal population effort which is substantially below the average population effort.

The grey bands in Figure 4 illustrate the results derived in Proposition 2(iii) that beliefs of all agents slowly converge to the lowest effort exerted in the first period of the games when there is no social influence in the network.

\subsection{Effects of Social Network Topology}

Previous work on opinion formation (e.g. Golub and Jackson (2009), Acemoglu et al. (2010)) has demonstrated the importance of the social network structure on the emergence of consensus in a population and the ability to learn the true state of the world. In this section we investigate how the ability of a population to coordinate on an effort level in our minimum effort game and the efficiency of the emerging effort level is influenced by different properties of the social network.

\section{Number of Links}

First we explore the effect of a changing level of connectedness in the network by varying the parameter $p$ which determines the probability that there is a link between two nodes. As can be seen in Figure 5 increasing this parameter has a non-linear effect on the distribution of the emerging long-run effort levels. In particular, the change in $p$ has a significant positive effect only on a short interval from 0.1 to 0.2 . Further increase in the number of links doesn't affect the expectations and thus the long-run effort levels in a statistically significant way. Hence, above a certain minimal level a higher degree of connectedness in the network does not foster the emergence of more efficient equilibria with higher effort level. 


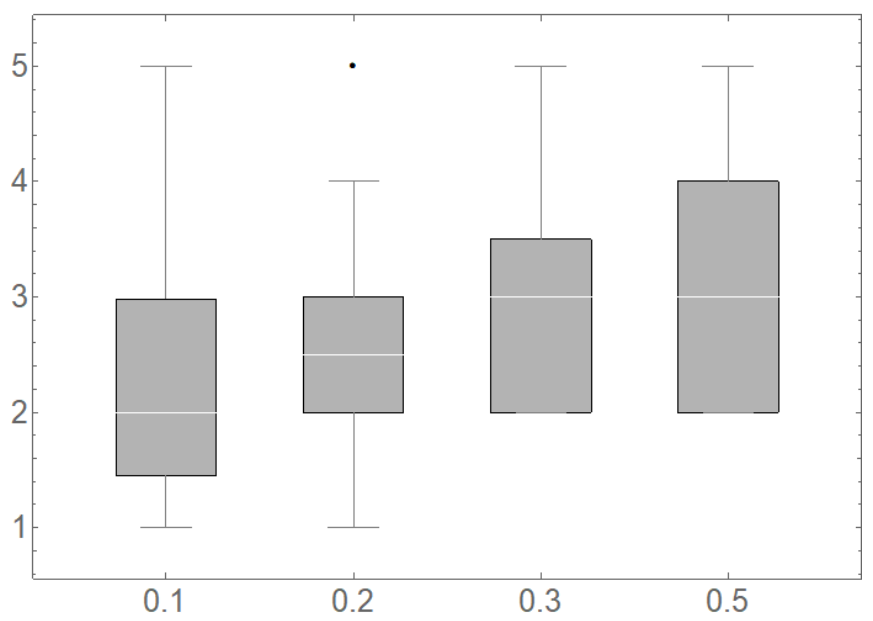

Figure 5: Distribution of average efforts at $t=40$ for linking probability $p=0.1,0.2,0.3,0.5$.

\section{Network Centralization}

So far we have considered random networks with identical linking probabilities between all agents. However, many social networks are characterized by a 'core-periphery' structure where a few central agents are each linked to a large number of individuals (see Borgatti and Everett (1999)). In order to study the effect of such (partially) centralized communication structure, we first consider the extreme case of a star network, in which all nodes are connected to one central agent, and then consider scenarios with several coexisting star networks.

Contrary to the weak effect of increasing connectedness in the random network, changing the type of the network into a star network with a single center boosts the expected minimum effort and the payoffs in the long run. Comparing the individual expectations and variances for random and star networks in Figures 1 and 6 one can observe that the expectations about the minimum effort converge to much higher level when the network is a 


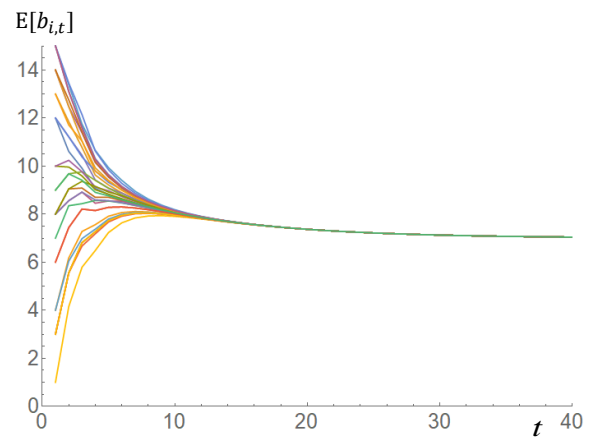

(a)

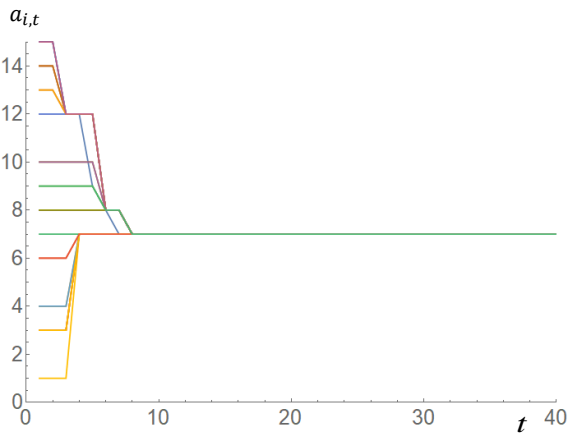

(b)

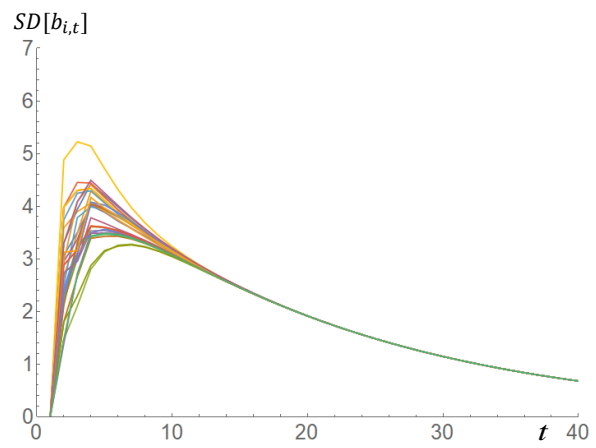

(c)

Figure 6: Dynamics of expected values of minimal effort (a), chosen actions (b) and variance of the belief distributions for all agents in a single run with a star social network.

star. The significant positive effect from this change in network topology on the final distribution of average efforts is shown in Figure 7. However, the variance of this distribution is much higher in the case of a star network. This is implied by the strong dependence of the outcome on the initial belief of the central node in the star. More specifically, each of the periphery nodes in the network learns about the belief of the central node, which over time then is adapted to the average beliefs of all periphery nodes. If an agent with pessimistic initial beliefs is located at the center of the star and 


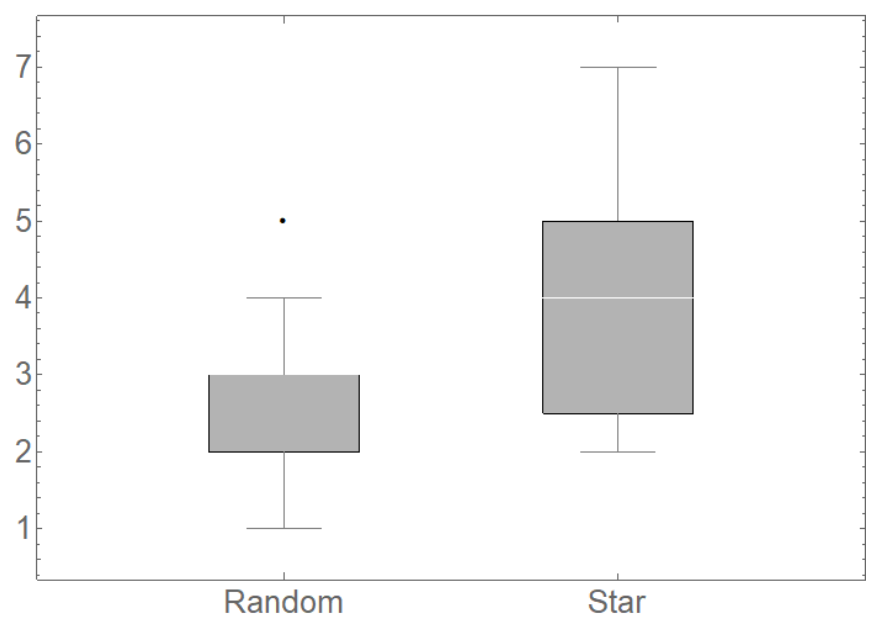

Figure 7: Distribution of average efforts at $t=40$ for a random network (left) and a star network (right)

therefore the only source of belief communication for periphery nodes, the average beliefs in the network quickly become more pessimistic. While a very optimistic center will push the beliefs in the network up.

As becomes apparent from Figure 6, the beliefs of the different agents become uniform much faster in a centralized network, which leads to a faster convergence of actions. Since average effort levels exhibit a negative trend as long as there is substantial heterogeneity of beliefs in the population, the fast convergence of beliefs leads to systematically higher effort levels compared to the random network. Given this effect of centralization, higher confidence in beliefs of social contacts for a large range of $\chi$ has a stronger effect in the star network compared to the random one (Figure 8). The increase of $\chi$ in a star network however has a significant positive effect only up to some level. Increasing the value of $\chi$ from 0.6 to 0.9 affects the long run distribution of beliefs negatively (see Figure 8).

If the centralization of the information flow is not global, like in a network 


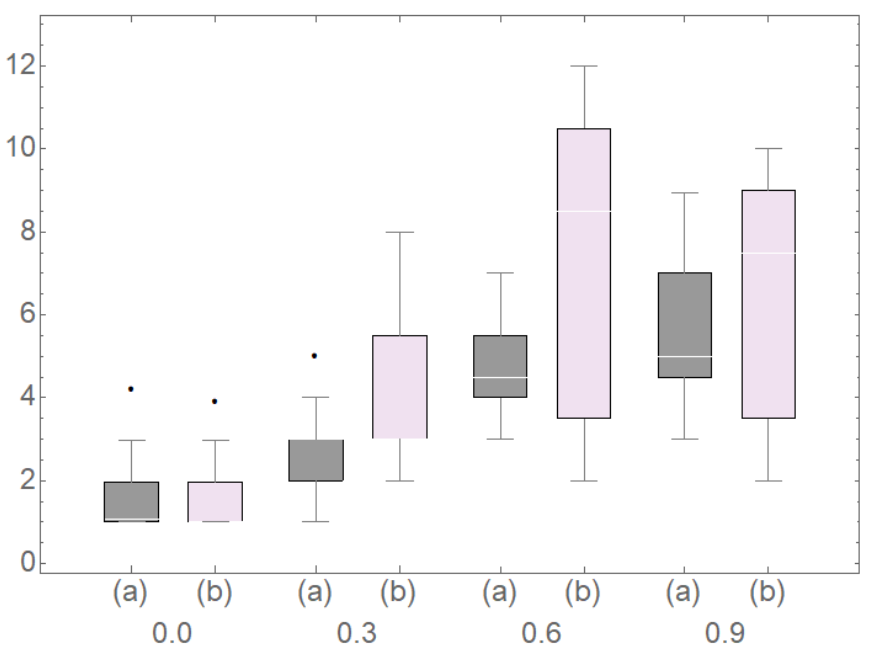

Figure 8: Distribution of average efforts at $t=40$ with a random network (a) and a star network (b) for $\chi=0,0.3,0.6,0.9$.

with a single star, but rather characterized by the coexistence of several influential 'local stars' the positive effect of the network centralization quickly diminishes. Figure 9 shows the distribution of long-run efforts for networks with one to six star components. Increasing the number of components from two to three and again from three to four components each yields a significant reduction in long-run effort, and the distribution of effort in a network with four star components is actually already below the distribution under a random network. Figure 10 illustrates the dynamics of individual beliefs and actions for a network with three star components. The mechanism leading to the relatively low long-term effort can be clearly identified in this figure. Individual beliefs of agents in each of the three components converge quickly due to communication of beliefs and social influence, however, agents from each component are repeatedly matched in groups with agents from the component with the lowest beliefs, yielding low effort observations for these agents. Hence, beliefs in all components over time slowly adjust 


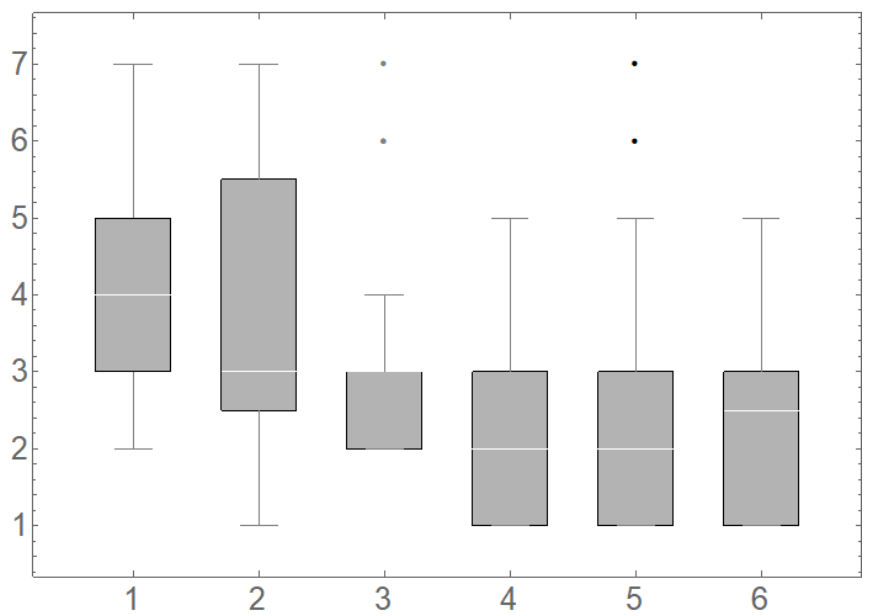

Figure 9: Distribution of average efforts at $t=40$ for star network (left) and segregated networks with 2 to 6 star components.

downwards towards the beliefs of the lowest component and actions across these components converge to a level determined by the actions taken by members of the most pessimistic component.

The effect of a segregation of the network into several disconnected components is much weaker if each component is a random network. Simulation results not reported in detail here show that increasing the number of components in general has only insignificant effects in such a setting.

Before analyzing an extension of our benchmark model, we like to point out that in all simulation runs in all settings considered in this section the beliefs and effort choices eventually become uniform. In particular, this observation also holds true for scenarios with high values of the social influence parameter $\chi$. Relating this to Proposition 3, which shows that for large values of that parameter there always exist social networks and initial beliefs such that convergence to a uniform profile does not occur, shows that the types of social networks that do not induce uniform long-run profiles are of 


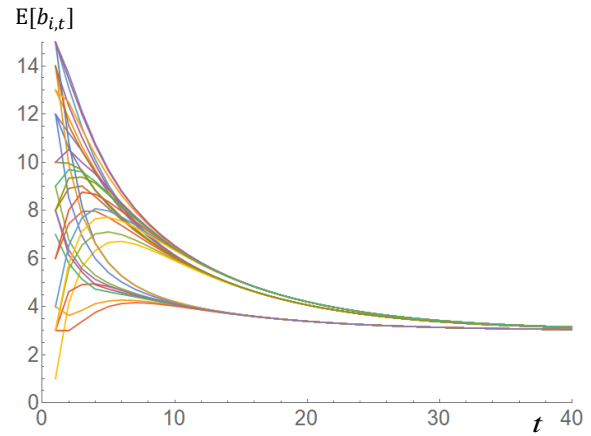

(a)

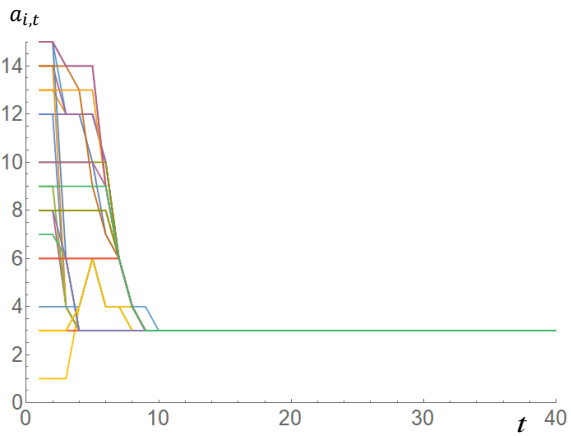

(b)

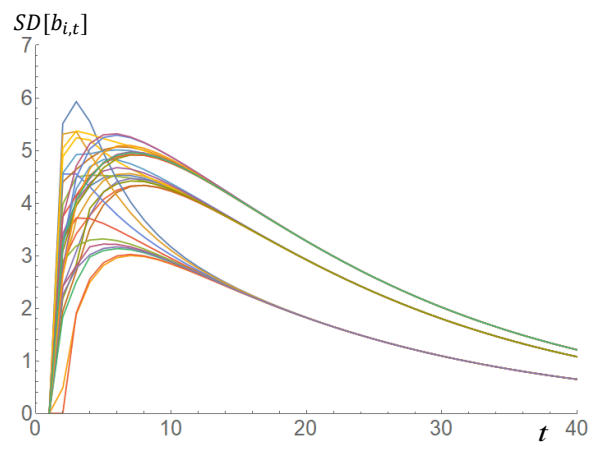

(c)

Figure 10: Dynamics of expected values of minimal effort (a), chosen actions (b) and variance of the belief distributions (c) for individual agents in a single run with a segregated social network with 3 star components.

very special structure. As sketched in Section 3, long-run heterogeneity of beliefs requires the existence of separated components in the network where the social influence in the component with optimistic beliefs is sufficiently strong to outweigh the low-effort observations made by the agents in that component who are matched with members of the (smaller) component with pessimistic beliefs. Simulation results not reported here show that adding a single connection between these components typically is sufficient to induce uniform long-run beliefs in the population. Also, the results reported in 
the previous paragraphs show that even under fully separated components uniform beliefs and effort choices emerge in the long-run, if the components are of equal size.

\section{Communication of Information}

In our benchmark model we assume that an agent receives information about the outcomes of the minimal effort games in groups other than their own only indirectly through the communication of the beliefs of their social contacts. However, in many situations, individuals might not only communicate their beliefs to their social contacts, but also the actual outcome of their own interaction group. In this section we analyze whether the presence of such communication of information about the group outcomes in the social network changes the qualitative effects of belief communication. Furthermore, we explore whether communication of information might act as a substitute for the communication of beliefs or whether it might even reinforce the (positive) effect of belief communication on the long-run effort level emerging in the population.

We extend the model described in section 2 by assuming that, when building their intermediate beliefs, agents do not only take into account the outcome of their own interaction group, but also that in all groups of their social contacts. In particular, we replace in equation (1) term $\mathbb{1}_{\underline{e}_{i, t}}$ with $\hat{b}_{i, t}(e)$, which is given by

$$
\hat{b}_{i, t}=\mathbb{1}_{\underline{e}_{i, t}}+\kappa \frac{1}{\eta_{i}} \sum_{j \in m_{i}(s)}\left(\mathbb{1}_{\underline{e}_{j, t}}-\mathbb{1}_{\underline{e}_{i, t}}\right) .
$$

Here $0 \leq \kappa \leq 1$ is a weight that the agent $i$ assigns to the information about the minimum effort observed and communicated by her social contacts. Thus, we obtain the following generalization of equation (1), describing the intermediate belief formation with information communication: 


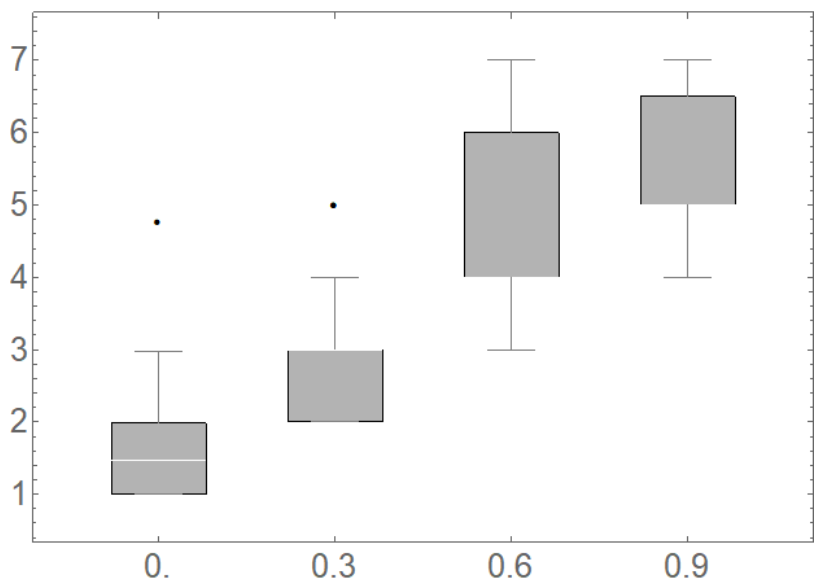

Figure 11: Distribution of average efforts at $t=40$ for $\chi=0,0.3,0.6,0.9$, with $\kappa=0.3$.

$$
\tilde{b}_{i, t+1}(e)=(1-\xi) b_{i, t}(e)+\xi \hat{b}_{i, t}(e)
$$

with $\hat{b}_{i, t}$ given in (3). For $\kappa=0$ this formulation gives our benchmark model studied in the previous section.

We again use our baseline parametrization and additionally set $\kappa=0.3$ as the baseline value for the weight assigned to the observations of the social contacts. Figure 11 shows that the impact of variation of the confidence parameter $\chi$ on the long run effort choice stays significant also with communication of information. Comparing the figure with Figure 2 also shows that quantitatively the effects of an increas of the social influence parameter $\chi$ are hardly affected by the presence of communication of information. Extensive analyses of the extended model for all the scenarios examined in Section 4 furthermore show that all earlier results remain qualitatively unchanged also in the presence of information communication. Statistical tests demonstrating this are presented in Appendix B together with the tests for 


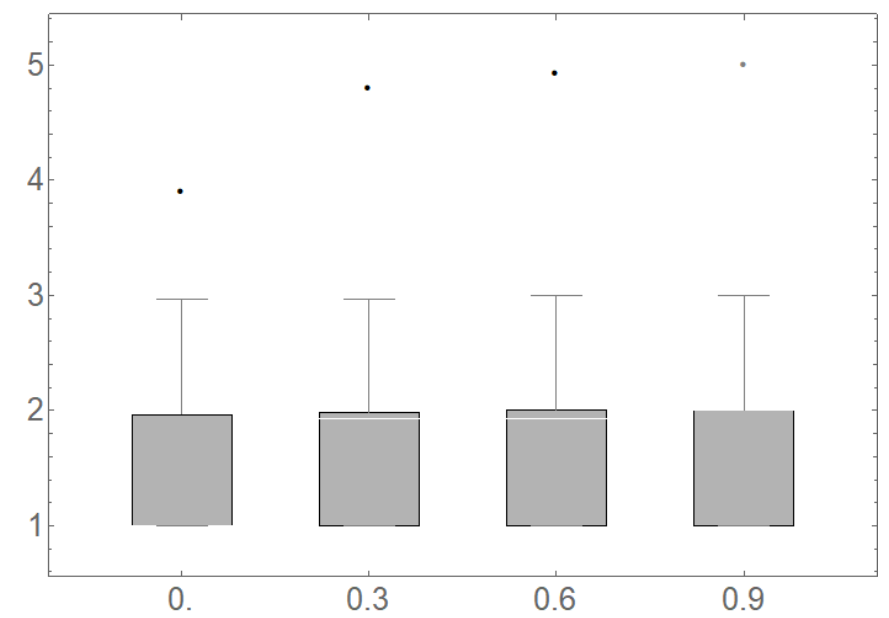

Figure 12: Distribution of average efforts at $t=40$ for $\kappa=0,0.3,0.6,0.9$, when $\chi=0$.

the baseline model.

Concerning the question whether the exchange of information can act as a substitute for the exchange of beliefs, we show in Figure 12 how the distribution of long-run effort changes if there is no exchange of beliefs $(\chi=0)$ and the parameter $\kappa$ determining the weight of the information obtained from an agent's social contacts is increased. The figure clearly demonstrates that exchanging only information without exchanging beliefs has hardly any positive effect on the level of long-run effort which emerges. This insight is consistent with the intuition developed above that the main role of the exchange of beliefs is that agents in this way get signals about (expected) minimal effort in groups that are more positive than the actual project outcomes which are observed. This role cannot be played by the communication of actual minimal efforts in the groups of the social contacts. Therefore, the communication of information about project outcomes cannot act as a substitute for the communication of beliefs in fostering more efficient outcomes 
of the minimal effort game. Overall, our results show that the main results discussed in the previous section qualitatively stay intact if apart from beliefs also information is communicated in the social network.

\section{Conclusions}

This paper highlights from a theoretical perspective the potential importance of social influence for improving efficiency of the outcome of group production problems with strategic complementarities. Also, it shows that the topology of the social network has a significant influence on the achieved outcome. Unfortunately, at this point experimental studies exploring the role of social influence in such a setting is missing. Hence, the analysis provided in this paper is a natural basis for designing and carrying out experiments clarifying in how far the effects identified in our study are also observable in the lab. Also from a theoretical perspective it would be interesting to explore the relevance of several of the assumptions that have been made in this paper. This includes the consideration of endogenous updating of the social network as well a generalization of the agents' behavior by incorporating logit best reply or social preferences or a more refined expectation updating process, e.g. like the one developed in Grimm and Mengel (2019) based on its good match with experimental evidence. These issues are left for future research.

\section{Acknowledgments}

This work has received funding from the European Union's Horizon 2020 research and innovation programme under the Marie Sklodowska-Curie grant agreement No 721846 "Expectations and Social Influence Dynamics in Economics" (ExSIDE). We are grateful for helpful comments from Yan Chen, Anna Mauleon, Vincent Vannetelbosch and audiences at Bielefeld Univer- 
sity, Université catholique de Louvain, the Annual Conference on Computing in Economics and Finance 2018 (Milano), the Annual Meetings of the Armenian Economic Association 2018 (Yerevan), the Lisbon Meetings in Game Theory and Applications 2018 and the GSE Summer Forum on Computational and Experimental Economics 2019 (Barcelona). 


\section{Appendix A. Proofs}

Proof of Proposition 1. We first show a Lemma, which will be used in this proof as well as in the proofs of the following propositions.

Lemma 1. Consider beliefs $b^{1}, b^{2} \in \Delta(\mathcal{X})$ such that $a^{*}\left(b^{1}\right) \leq a^{*}\left(b^{2}\right)$ then $a^{*}\left(\kappa b^{1}+(1-\kappa) b^{2}\right) \in\left[a^{*}\left(b^{1}\right), a^{*}\left(b^{2}\right)\right]$ for all $\kappa \in[0,1]$.

Proof of Lemma 1. In light of the form of $\pi\left(e_{i}, e_{-i}\right)$ we can write the expected profit of an agent with effort $e$ and belief $b$ as

$$
\pi^{e}(e, b)=\alpha\left(\sum_{\tilde{e} \leq e} b(\tilde{e}) \tilde{e}+e \sum_{\tilde{e}>e} b(\tilde{e})\right)-\beta e .
$$

Hence,

$$
\Delta \pi^{e}(e, b)=\pi^{e}(e+1, b)-\pi^{e}(e, b)=\alpha \sum_{\tilde{e}>e} b(\tilde{e})-\beta,
$$

which is (weakly) decreasing in $e$. Now consider some effort $=e^{1}<a^{*}\left(b^{1}\right)$. Since $e^{1}<a^{*}\left(b^{1}\right) \leq a^{*}\left(b^{2}\right)$ and $\Delta \pi(e, b)$ is decreasing in $e$, we must have $\Delta \pi^{e}\left(e^{1}, b^{1}\right) \geq 0$ and $\Delta \pi^{e}\left(e^{1}, b^{2}\right) \geq 0$. Therefore,

$$
\Delta \pi^{e}\left(e^{1}, \kappa b^{1}+(1-\kappa) b^{2}\right)=\kappa \Delta \pi^{e}\left(e^{1}, b^{1}\right)+(1-\kappa) \Delta \pi^{e}\left(e^{1}, b^{2}\right) \geq 0 .
$$

Since $a^{*}\left(\kappa b^{1}+(1-\kappa) b^{2}\right)$ is the largest effort among those maximizing the expected profit of the agent, this shows that $e_{1}<a^{*}\left(\kappa b^{1}+(1-\kappa) b^{2}\right)$. Hence $a^{*}\left(\kappa b^{1}+(1-\kappa) b^{2}\right) \geq a^{*}\left(b_{1}\right)$. Analogous arguments show that $a^{*}\left(\kappa b^{1}+(1-\right.$ $\left.\kappa) b^{2}\right) \leq a^{*}\left(b_{2}\right)$. This completes the proof of the lemma.

To prove claim (i) of the proposition consider a profile $B_{t}$ such that $a^{*}\left(b_{i, t}\right)=e$ for all $i \in N$. It should be noted that under such a profile $\underline{e}_{i, t}=e$ for all $i \in N$ regardless of the realization of the group partition. Hence $B_{t+1}$ is deterministic and we show that also for $B_{t+1}$ we have $a^{*}\left(b_{i, t+1}\right)=e$ for all $i \in N$. Claim (i) then follows by induction. To show that $a^{*}\left(b_{i, t+1}\right)=e$ for 
all $i \in N$, we consider an arbitrary agent $i$. Since $\underline{e}_{i, t}=e$ and $a^{*}\left(\mathbb{1}_{e}\right)=e$, it follows from Lemma 1 that

$$
a^{*}\left(\tilde{b}_{i, t+1}\right)=a^{*}\left((1-\xi) b_{i, t}+\xi \mathbb{1}_{e}\right)=e .
$$

Since the same reasoning also applies to all social contacts of $i$, repeated application of the lemma establishes that $a^{*}\left(\frac{1}{\eta_{i}(s)} \sum_{j \in m_{i}(s)} \tilde{b}_{j, t+1}\right)=e$, and therefore

$$
a^{*}\left(b_{i, t+1}\right)=a^{*}\left((1-\chi) \tilde{b}_{i, t}+\chi \frac{1}{\eta_{i}(s)} \sum_{j \in m_{i}(s)} \tilde{b}_{j, t+1}\right)=e .
$$

Hence, we obtain part (i) of the proposition.

To show part (ii) we define for some arbitrary effort $\hat{e} \in \mathcal{X} \backslash\{e\}$ the maximal probability for this effort level in any belief distribution in $B_{\tau}$ as $\bar{b}(\hat{e})_{\tau}=\max \left[b_{i, \tau}(\hat{e}): i \in N\right]$ for all $\tau \geq t$. Since $\underline{e}_{j, \tau}=e \neq \hat{e}$ for all $j \in N$ and $\tau \geq t$ we have

$$
\tilde{b}_{j, \tau+1}(\hat{e})=(1-\xi) b_{j, \tau}(\hat{e}) \leq(1-\xi) \bar{b}(\hat{e})_{\tau}
$$

and therefore

$$
b_{i, \tau+1}(\hat{e})=(1-\xi)(1-\chi) b_{i, \tau}(\hat{e})+\frac{\chi}{\eta_{i}(s)} \sum_{j \in m_{i}(s)} \tilde{b}_{j, \tau+1} \leq(1-\xi) \bar{b}(\hat{e})_{\tau}
$$

for all $i \in N$. Hence, $\bar{b}(\hat{e})_{\tau+1} \leq(1-\xi) \bar{b}(\hat{e})_{\tau}$ and accordingly $\lim _{\tau \rightarrow \infty} \bar{b}(\hat{e})_{\tau}=$ 0 for all $\hat{e} \neq e$. This implies that $b_{i, \tau} \rightarrow \mathbb{1}_{e}$ for $\tau \rightarrow \infty$ for all $i \in N$

Proof of Proposition 2. (i) By definition we have $\underline{e}_{i, t} \leq a^{*}\left(b_{i, t}\right)$ for all $i$ and all $t$. Hence, $a^{*}\left(\mathbb{1}_{\underline{e}_{i, t}}\right)=\underline{e}_{i, t} \leq a^{*}\left(b_{i, t}\right)$. Using Lemma 1 we obtain

$$
a_{i, t+1}=a^{*}\left(b_{i, t+1}\right)=a^{*}\left((1-\xi) b_{i, t}+\xi \mathbb{1}_{\underline{e}_{i, t}}\right) \leq a^{*}\left(b_{i, t}\right)=a_{i, t} .
$$

(ii) First, we show that for any agent $i$ with $a_{i, 1}=\underline{e}_{1}:=\min _{j \in N} a_{j, 1}$ we must have $a_{i, \tau}=\underline{e}_{\tau}=\underline{e}_{1} \forall \tau \geq 1$. This can be shown by induction. Assume 
that for all $\tau=1, . ., t$ beliefs $b_{i, \tau}$ are such that $a^{*}\left(b_{i, \tau}\right)=\underline{e}_{t}=\underline{e}_{1}$. Then obviously we have $a_{i, t}=\underline{e}_{1}$ and therefore, under consideration of $\chi=0$, the updated belief reads

$$
b_{i, t+1}=\tilde{b}_{i, t+1}=(1-\xi) b_{i, t}+\xi \mathbb{1}_{\underline{e}_{1}} .
$$

Using Lemma 1 it follows from $a^{*}\left(b_{i, t}\right)=a^{*}\left(\mathbb{1}_{\underline{e}_{1}}\right)=\underline{e}_{1}$ that $a_{i, t+1}=a^{*}\left(b_{i, t+1}\right)=$ $\underline{e}_{1}$. For any agent $j \neq i$ we have $a^{*}\left(b_{j, t}\right) \geq \underline{e}_{1}$ and $\underline{e}_{j, t} \geq \underline{e}_{1}$, which implies that

$$
a_{j, t+1}=a^{*}\left((1-\xi) b_{j, t}+\xi \mathbb{1}_{\underline{e}_{j, t}}\right) \geq \underline{e}_{1} .
$$

From this we conclude that $\underline{e}_{t+1} \geq \underline{e}_{1}$, which in light of $a_{i, t+1}=\underline{e}_{1}$ implies $\underline{e}_{t+1}=\underline{e}_{1}$. This completes the induction and we have shown that $a_{i, t}=\underline{e}_{t}=$ $\underline{e}_{1}$ for all $t \geq 1$.

Consider now an agent $j$ with $a_{j, t}>\underline{e}_{1}$. Taking into account that $e$ is a strictly optimal action under belief $b=\mathbb{1}_{e}$ and the continuity of $\pi^{e}$ with respect to $b$, it follows that there exists some $\tilde{\lambda}$ such that $a^{*}\left(\lambda \mathbb{1}_{\underline{e}_{1}}+(1-\right.$ $\left.\lambda) b_{j, t}\right)=\underline{e}_{1}$ for all $\lambda \geq \tilde{\lambda}$. Choose a $\tilde{T}$ such that $(1-\xi)^{\tilde{T}}<1-\tilde{\lambda}$. There is a positive probability that agent $j$ is matched with an agent $i$ with $a_{i, t}=\underline{e}_{1}$ for $\tilde{T}$ periods in a row. The belief of agent $j$ in period $t+\tilde{T}$ then is given by

$$
b_{j, t+\tilde{T}}=(1-\xi)^{\tilde{T}} b_{j, t}+\left(1-(1-\xi)^{\tilde{T}}\right) \mathbb{1}_{\underline{e}_{1}}
$$

and our reasoning above shows that, in case such a matching pattern occurs, this agent $j$ switches to action $a_{j, t+\tilde{T}}=\underline{e}_{1}$. Following the arguments provided in the first part of the proof, this implies that $a_{j, \tau}=\underline{e}_{1}$ for all $\tau \geq t+\tilde{T}$. The same reasoning can be applied sequentially to every agent $j$ with $a_{j, t}>\underline{e}_{1}$. Together, this establishes that from every profile of beliefs $B_{t}$, that can be reached with positive probability from $B_{1}$, there is a positive probability path to a profile $\tilde{B}$ with the property that $a^{*}\left(\tilde{b}_{i}\right)=\underline{e}_{1}$ for all $i \in N$. Denote the set of all such belief profiles $\tilde{B}$ by $\tilde{\mathcal{B}}=\left\{\tilde{B} \mid a^{*}\left(\tilde{b}_{i}\right)=\underline{e}_{1} \forall i \in N\right\}$. We know from Proposition 1 that this set $\tilde{\mathcal{B}}$ 
of belief profiles is absorbing. Together with the fact that there is a transition path with positive probability from any reachable state into $\tilde{\mathcal{B}}$, this implies that $\mathbb{P}\left(B_{t} \notin \tilde{\mathcal{B}}\right) \rightarrow 0$ for $t \rightarrow \infty$. This shows part (ii) of the proposition. Part (iii) follows directly from the observation that if $B_{t} \in \tilde{\mathcal{B}}$ then $b_{i, t+\tau}(\hat{e}) \leq(1-\xi)^{\tau} b_{i, t}(\hat{e})$ for all $i \in N, \hat{e} \neq \underline{e}_{1}$.

Proof of Proposition 3. Consider any initial belief profile $B_{1}$ with $\mid\{i \in N$ : $\left.a^{*}\left(b_{i, 1}\right)=\underline{e}_{1}\right\} \mid=1$. Without restriction of generality we label the agent with the smallest initial effort as agent 1 . Hence, we have $a^{*}\left(b_{1,1}\right)=\underline{e}_{1}$ and $a^{*}\left(b_{i, 1}\right)>\underline{e}_{1} \forall i=2, . ., n$. Consider the social network $s$ with $m_{1}(s)=$ $\emptyset, m_{i}(s)=N \backslash\{1, i\}$. Since agent 1 does not have any social contacts under this social network, the beliefs of agent 1 in period $t$ are given by

$$
b_{1, t}=(1-\xi)^{t-1} b_{1,1}+\left(1-(1-\xi)^{t-1}\right) \mathbb{1}_{\underline{e}_{1}}
$$

and it follows from Lemma 1 that $a_{1, t}=\underline{e}_{1}$ for all $t$.

Considering agents $i=2, . ., n$ we proceed by induction. Assume that $a^{*}\left(b_{i, \tau}\right)>\underline{e}_{1}$ for all $i=2, . . n$ and $\tau=1, . ., t$. We show that then also $a^{*}\left(b_{i, t+1}\right)>\underline{e}_{1}$ for all $i=2, . . n$. Define, as in the proof of Proposition 2, the expected profit difference between two adjacent effort levels as $\Delta \pi^{e}(e, b)=$ $\pi^{e}(e+1, b)-\pi^{e}(e, b)$. Since $\Delta \pi^{e}(e, b)$ is weakly decreasing in $e$ and $a^{*}\left(b_{i, t}\right)>$ $\underline{e}_{1}$ for all $i=2, . ., n$ it follows that

$$
\Delta \pi^{e}\left(\underline{e}_{1}, b_{i, t}\right)=\alpha \sum_{\tilde{e}>\underline{e}_{1}} b_{i, t}(\tilde{e})-\beta \geq 0
$$

Defining $x_{i, t}=\sum_{\tilde{e}>\underline{e}_{1}} b_{i, t}(\tilde{e})$, it follows that

$$
x_{i, t} \geq \frac{\beta}{\alpha} \forall i=2, . ., n .
$$

In every period $(k-1)$ agents are matched with agent 1 and hence observe $\underline{e}_{i, t}=\underline{e}_{1}$, whereas for the remaining $n-k$ agents we have $\underline{e}_{i, t}>\underline{e}_{1}$. Denote by agent $j$ one of the agents matched with agent 1 in $t$. Furthermore, denote 
by $y_{t}=\sum_{i \neq 1, j} \sum_{\tilde{e}>\underline{e}_{1}} b_{i, t}(\tilde{e})$ the sum of the probabilities that all agents apart from agents 1 and $j$ put on effort choices above $\underline{e}_{1}$. Clearly, we have $y_{t} \geq(n-2) \frac{\beta}{\alpha}$. Defining $\tilde{x}_{j, t}=\sum_{\tilde{e}>\underline{e}_{1}} \tilde{b}_{j, t}(\tilde{e})$ we obtain from (1)

$$
\tilde{x}_{j, t+1}=(1-\xi) x_{j, t} .
$$

Furthermore, we define by $\tilde{y}_{t}=\sum_{i \neq 1, j} \sum_{\tilde{e}>\underline{e}_{1}} \tilde{b}_{i, t}(\tilde{e})$ and we get

$$
\tilde{y}_{t+1}=(1-\xi) y_{t}+\xi(n-k)
$$

Inserting this into the belief adjustment due to social influence for agent $j$ (see 2) we obtain

$$
\begin{aligned}
x_{j, t+1} & =(1-\chi) \tilde{x}_{j, t+1}+\frac{\chi}{n-2} \tilde{y}_{t+1} \\
& =(1-\chi)(1-\xi) x_{j, t}+\frac{\chi}{n-2}\left((1-\xi) y_{t}+\xi(n-k)\right) \\
& \geq(1-\chi)(1-\xi) \frac{\beta}{\alpha}+\frac{\chi}{n-2}\left((1-\xi)(n-2) \frac{\beta}{\alpha}+\xi(n-k)\right) \\
& =(1-\xi) \frac{\beta}{\alpha}+\chi \frac{n-k}{n-2} \xi
\end{aligned}
$$

where we have used that $x_{j, t}>\frac{\beta}{\alpha}$ and $y_{t} \geq(n-2) \frac{\beta}{\alpha}$. If $\chi>\underline{\chi}$ this directly implies that $x_{j, t+1} \geq \frac{\beta}{\alpha}$ and therefore $a^{*}\left(b_{j, t+1}\right)>\underline{e}_{1}$. Clearly, the beliefs of all agents who have not been matched with agent 1 in period $t$ are more optimistic than that of agent $j$ and therefore we have $a^{*}\left(b_{i, t+1}\right)>\underline{e}_{1}$ for all $i=2, . ., n$. This completes the induction. Overall, we have shown that for all agents $i=2, . ., n$ we have $a_{i, t}>\underline{e}_{1}$ for all $t \geq 1$. Together with $a_{1, t}=\underline{e}_{1}$ for all $t \geq 1$ this shows that with probability one $\max _{i \in N}\left[a_{i, t}\right]<$ $\underline{e}_{1}=\min _{i \in N}\left[a_{i, t}\right]$, which completes the proof.

\section{Appendix B. Test Results}

We perform Wilcoxon signed-rank test to show the significance of difference between distributions of long run effort choices. Tables 1-7 in this appendix 
show the $p$-values of the tests. ${ }^{8}$

Table 1: Effect of the social influence on long-run efforts.

\begin{tabular}{lccc}
\hline \hline$\chi$ & 0 and 0.3 & 0.3 and 0.6 & 0.6 and 0.9 \\
\hline$\kappa=0$ & 0.0002 & 0.0001 & 0.0006 \\
$\kappa=0.3$ & 0.0001 & 0.0001 & 0.0457 \\
\hline \hline
\end{tabular}

The table shows the effects of increasing social influence on the long-run efforts in the baseline model $(\kappa=0)$ and the extended model with the information communication $(\kappa=0.3)$ with random network.

We compare each pair of long-run effort distributions for scenarios with trust parameter values $\chi$ equal to 0 and $0.3,0.3$ and $0.6,0.6$ and 0,9 .

Table 2: Effect of changing the random network connectedness.

\begin{tabular}{lccc}
\hline \hline & 0.1 and 0.2 & 0.2 and 0.3 & 0.3 and 0.5 \\
\hline$\kappa=0$ & 0.0419 & 0.2396 & 0.4666 \\
$\kappa=0.3$ & 0.6677 & 0.7794 & 0.5882 \\
\hline \hline
\end{tabular}

The table shows the effect of increasing average degree in the random network. We compare the pairs of long-run effort distributions in random networks with probabilities of link formation p equal to 0.1 and $0.2,0.2$ and 0.3 , 0.3 and 0.5 . The Wilcoxon signed-rank tests are performed for the baseline model $(\kappa=0)$ and the extended model with the information communication $(\kappa=0.3)$.

\footnotetext{
${ }^{8}$ For the tests in Table 1 and further we consider the difference of two distributions to be significant when $p<0.05$.
} 
Table 3: Social influence effect in the star network.

\begin{tabular}{lccc}
\hline \hline$\chi$ & 0 and 0.3 & 0.3 and 0.6 & 0.6 and 0.9 \\
\hline$\kappa=0$ & 0.0001 & 0.0012 & 0.0178 \\
$\kappa=0.3$ & 0.0001 & 0.0021 & 0.0021 \\
\hline \hline
\end{tabular}

The table shows the effects of increasing social influence on the long-run efforts in the baseline model $(\kappa=0)$ and the extended model with the information communication $(\kappa=0.3)$ with star network.

We compare each pair of long-run effort distributions for scenarios with trust parameter values $\chi$ equal to 0 and $0.3,0.3$ and $0.6,0.6$ and 0,9 .

Table 4: Effect of network centralization.

\begin{tabular}{lcccc}
\hline \hline & $\chi=0$ & $\chi=0.3$ & $\chi=0.6$ & $\chi=0.9$ \\
\hline$\kappa=0$ & 0.2708 & 0.0007 & 0.0076 & 0.1978 \\
$\kappa=0.3$ & 0.0894 & 0.0003 & 0.0085 & 0.1506 \\
\hline \hline
\end{tabular}

The test result in the table indicate the significance of difference between long-run effort distributions of random network with benchmark value of link formation probability 0.2 and star network. The tests are performed for the baseline model $(\kappa=0)$ and the extended model with information communication $(\kappa=0.3)$. Alongside with the benchmark value of trust parameter $\chi=0.3$ other levels of social influence are tested. 
Table 5: Effect of increasing segregation level in random networks.

\begin{tabular}{lccccc}
\hline \hline & 1 and 2 & 2 and 3 & 3 and 4 & 4 and 5 & 5 and 6 \\
\hline$\kappa=0$ & 0.9553 & 0.4441 & 0.2396 & 0.0119 & 0.6677 \\
$\kappa=0.3$ & 0.9553 & 0.8373 & 0.8082 & 0.0966 & 0.1850 \\
\hline \hline
\end{tabular}

The table shows Wilcoxon signed-rank tests results of comparing long-run effort distributions of random networks with 1 component and 2 disconnected components, as well as random networks with 2 and 3, 3 and 4, 4 and 5 , and 5 and 6 disconnected components. The average degree in all networks is kept equal to the benchmark value.

Table 6: Effect of increasing segregation level in networks with star components.

\begin{tabular}{lccccc}
\hline \hline & 1 and 2 & 2 and 3 & 3 and 4 & 4 and 5 & 5 and 6 \\
\hline$\kappa=0$ & 0.4441 & 0.0545 & 0.0239 & 0.5883 & 0.7795 \\
$\kappa=0.3$ & 0.0160 & 0.2396 & 0.0054 & 0.6407 & 0.6676 \\
\hline \hline
\end{tabular}

The table shows Wilcoxon signed-rank tests results of comparing long-run effort distributions of a star network and a network with 2 disconnected star components, networks with 2 and 3,3 and 4, 4 and 5, and 5 and 6 disconnected star components correspondingly.

\section{Appendix C. Robustness}

In this appendix we show the robustness of our model with respect to changes in size of the social network $(n)$, randomly generated groups' size $(k)$, the 
Table 7: Comparing the effects of $\chi$ and $\kappa$.

\begin{tabular}{lccc}
\hline \hline$\chi$ or $\kappa$ & 0 and 0.3 & 0.3 and 0.6 & 0.6 and 0.9 \\
\hline$\kappa=0$ & 0.0002 & 0.0001 & 0.0006 \\
$\chi=0$ & 0.0048 & 0.0594 & 0.0117 \\
\hline$\kappa=0.3$ & 0.0001 & 0.0001 & 0.0457 \\
$\chi=0.3$ & 0.2396 & 0.9256 & 0.1850 \\
\hline \hline
\end{tabular}

The table shows the effects of increasing social influence $(\chi)$ and coefficient of information communication $(\kappa)$ on the long-run efforts when the $\kappa$ and $\chi$ are set to 0.3 correspondingly.

number of possible strategies, that is, the highest effort level $(\bar{e})$, and the speed of beliefs' updating $(\xi)$. In particular, we show that the effect of the increasing level of trust in beliefs of social contacts remains the same when changing the benchmark parametrization.

\section{Network Size}

We compare the effect of increasing social influence in networks of sizes $n=15$ and $n=50$ to the benchmark of size 30 . We find that in smaller network of size 15 increasing the value of parameter $\chi$ from 0.6 to 0.9 doesn't have a significant effect (Figure $13^{9}$, Table 8). This results in a significant difference between distributions of long run efforts for network sizes 15 and 30 , the "cross-effect" of network size (Table $9, p=0.016$ ). In all remaining cases the increase in value of trust parameter $\chi$ has a significant effect on the long run effort choice, whereas the "cross-effect" of network size for a

\footnotetext{
${ }^{9}$ In this and further figures, the darker grey coloring of the plots is to represent the benchmark model.
} 
given value of $\chi$ is insignificant.

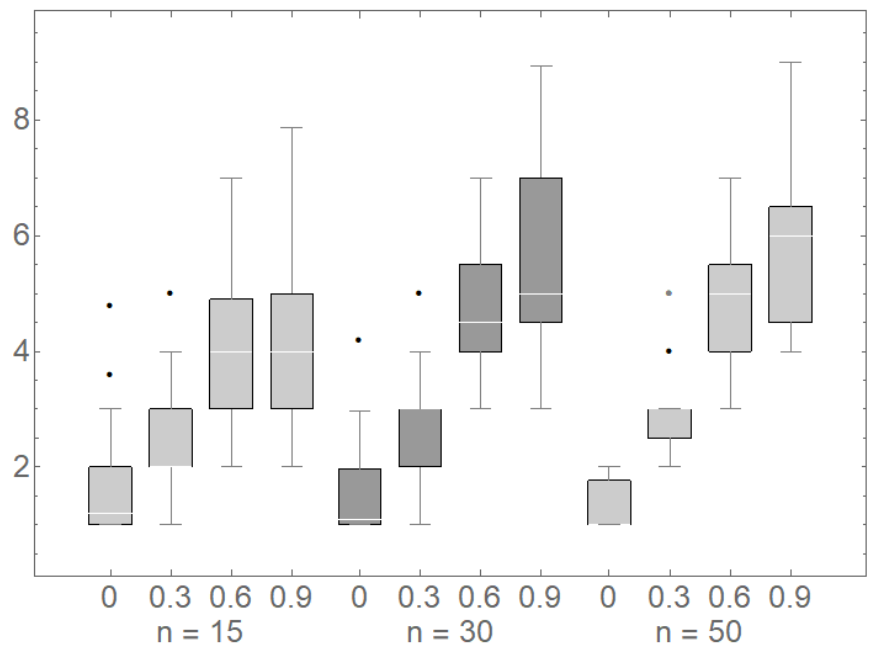

Figure 13: Distribution of average efforts at $t=40$ for $\chi=0,0.3,0.6,0.9$, for network sizes $n=15, n=30$ and $n=50$.

Table 8: Effect of increasing social influence on long-run efforts in different size networks.

\begin{tabular}{lccc}
\hline \hline$\chi$ & 0 and 0.3 & 0.3 and 0.6 & 0.6 and 0.9 \\
\hline$n=15$ & 0.0038 & 0.003 & 0.0545 \\
$n=30$ & 0.0002 & 0.0001 & 0.0006 \\
$n=50$ & 0.0001 & 0.0001 & 0.0018 \\
\hline \hline
\end{tabular}

The table shows the effect of increasing social influence on the long-run efforts in the baseline model $(\kappa=0)$. We compare each pair of long-run effort distributions for scenarios with trust parameter values $\chi$ equal to 0 and $0.3,0.3$ and $0.6,0.6$ and 0,9 when the size of the network is 15 , 30(benchmark) and 50 . 
Table 9: Cross-effect of the network size.

\begin{tabular}{lcc}
\hline \hline & 15 and 30 & 30 and 50 \\
\hline$\chi=0$ & 0.9256 & 0.1305 \\
$\chi=0.3$ & 0.9256 & 0.1851 \\
$\chi=0.6$ & 0.1213 & 0.5135 \\
$\chi=0.9$ & 0.016 & 0.6407 \\
\hline \hline
\end{tabular}

The table shows the "cross-effect" of increasing network size for different levels of social influence parameter $\chi$.

\section{Random Group Size}

The positive effect of the increasing $\chi$ holds when changing the size of random game groups $k$ in the network of size 30. This result is illustrated in Figure 14 and confirmed by test results in Table 10. We also find that the increasing the number of people in randomly formed groups playing minimum effort game decreases the efforts significantly. Thus, increase in $k$ from 2 to 5 , and from 6 to 10 leads to a significant decrease in long-run efforts for all analyzed values of the trust parameter. Moreover, the negative effect increasing the group size from 5 to 6 is significant given there is no social influence in the network (Table 11).

\section{Strategy Set}

For a given set of strategies $\mathcal{X}=\{1, \ldots, \bar{e}\}$ the positive effect of trust parameter on effort choice is independent from the value of $\bar{e}$ (Figure 15, Table 12). Despite of the difference in number of possible efforts and the higher average effort, the long-run effort' distribution doesn't differ significantly 


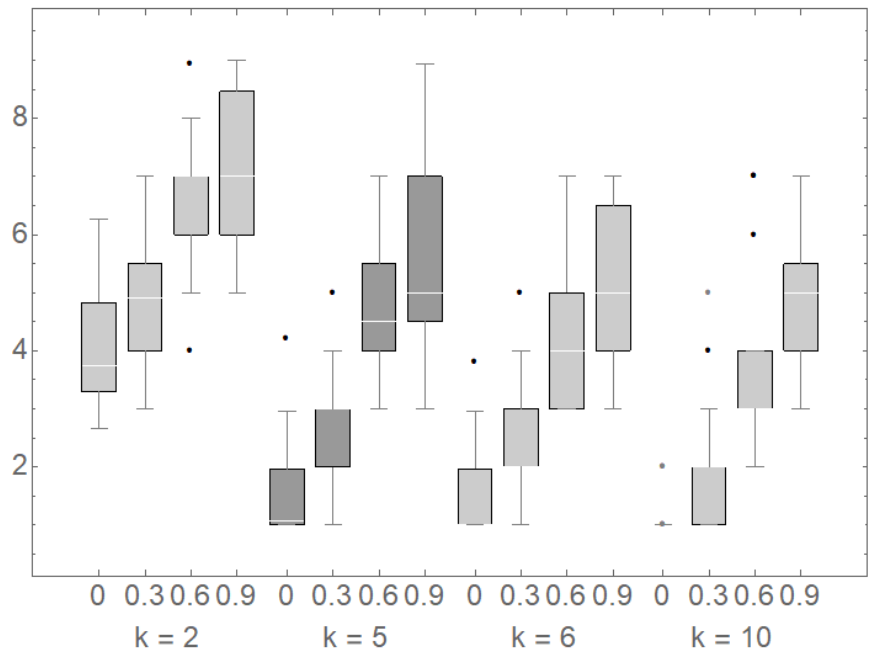

Figure 14: Distribution of average efforts at $t=40$ for random group sizes $k=2,5,6,10$ and $\chi=0,0.3,0.6,0.9$.

with absence of social influence. While this difference grows significantly when the trust parameter $\chi$ is positive (Table 13). This can be explained scaling up with the average of possible strategies.

\section{Speed of Updating}

Increasing the speed for updating old beliefs to new information affects the long-run effort choice for any level of trust in the network. The negative cross-effect of faster updating is significant (Table 15). Test results in Table 14 show that the positive effect from increasing social influence remains significant when $\xi=0.1$, and $\xi=0.3$. While for faster belief updating $(\xi=0.5, \xi=0.6)$ increase in trust parameter from absence of social influence $\chi=0$ to $\chi=0.3$ becomes insignificant. 
Table 10: Effect of increasing social influence on long-run efforts with different random group sizes.

\begin{tabular}{lccc}
\hline \hline$\chi$ & 0 and 0.3 & 0.3 and 0.6 & 0.6 and 0.9 \\
\hline$k=2$ & 0.0003 & 0.0001 & 0.0021 \\
$k=5$ & 0.0002 & 0.0001 & 0.0006 \\
$k=6$ & 0.0002 & 0.0001 & 0.0002 \\
$k=10$ & 0.0023 & 0.0001 & 0.0002 \\
\hline \hline
\end{tabular}

The table shows the effect of increasing social influence on the long-run efforts in the baseline model $(\kappa=0)$. We compare each pair of long-run effort distributions for scenarios with trust parameter values $\chi$ equal to 0 and $0.3,0.3$ and $0.6,0.6$ and 0,9 when the size of randomly generated groups $k$ are $2,5,6$, and 10 .

Table 11: Cross-effect of increasing the random group size.

\begin{tabular}{lccc}
\hline \hline & 2 and 5 & 5 and 6 & 6 and 10 \\
\hline$\chi=0$ & 0.0001 & 0.0458 & 0.0054 \\
$\chi=0.3$ & 0.0001 & 0.0826 & 0.0023 \\
$\chi=0.6$ & 0.0001 & 0.0646 & 0.0003 \\
$\chi=0.9$ & 0.0001 & 0.4009 & 0.0239 \\
\hline \hline
\end{tabular}

The table shows the "cross-effect" of increasing random group size $k$ for different levels of social influence parameter $\chi$. 


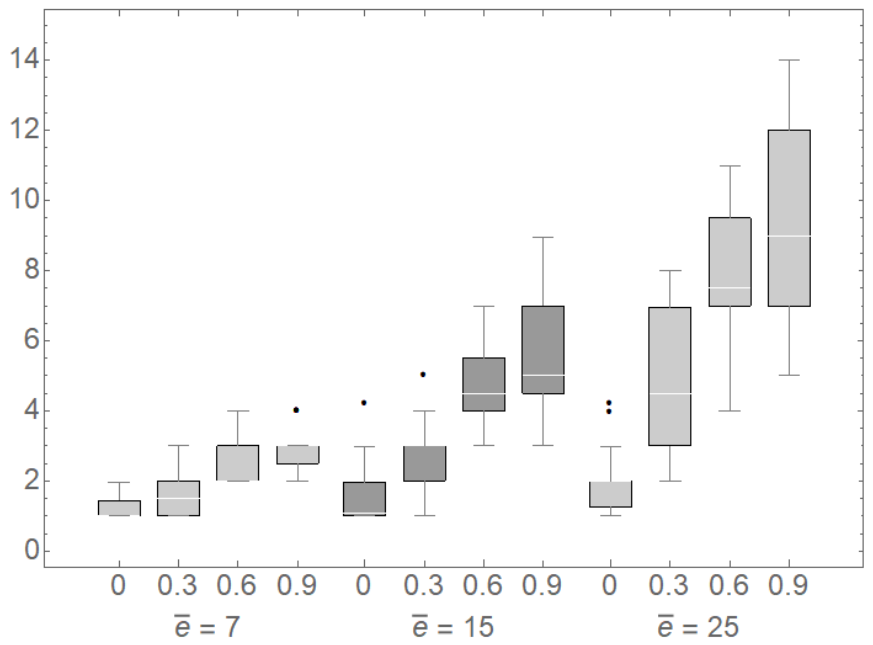

Figure 15: Distribution of average efforts at $t=40$ with highest possible effort and number of possible strategies $\bar{e}=7,15,25$ and $\chi=0,0.3,0.6,0.9$.

Table 12: Effect of increasing social influence on long-run efforts with different values of $\bar{e}$.

\begin{tabular}{lccc}
\hline \hline$\chi$ & 0 and 0.3 & 0.3 and 0.6 & 0.6 and 0.9 \\
\hline $\bar{e}=7$ & 0.0239 & 0.0002 & 0.0043 \\
$\bar{e}=15$ & 0.0002 & 0.0001 & 0.0006 \\
$\bar{e}=25$ & 0.0001 & 0.0001 & 0.0006 \\
\hline \hline
\end{tabular}

The table shows the effect of increasing social influence on the long-run efforts in the baseline model $(\kappa=0)$. We compare each pair of long-run effort distributions for scenarios with trust parameter values $\chi$ equal to 0 and $0.3,0.3$ and $0.6,0.6$ and 0,9 when the highest possible effort is 7,15 , and 25 . 
Table 13: Cross-effect of changing the highest possible effort.

\begin{tabular}{lcc}
\hline \hline & 7 and 15 & 15 and 25 \\
\hline$\chi=0$ & 0.0826 & 0.2111 \\
$\chi=0.3$ & 0.0068 & 0.0034 \\
$\chi=0.6$ & 0.0002 & 0.0007 \\
$\chi=0.9$ & 0.0002 & 0.0005 \\
\hline \hline
\end{tabular}

The table shows the "cross-effect" of increasing the highest possible effort $\bar{e}$ for different levels of social influence parameter $\chi$.

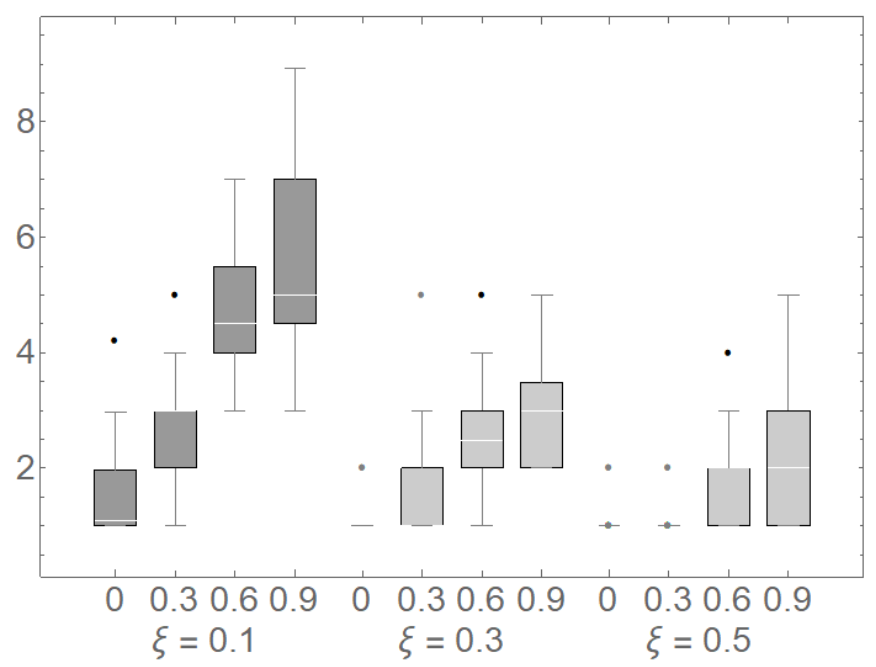

Figure 16: Distribution of average efforts at $t=40$ for $\chi=0,0.3,0.6,0.9$ with $\xi=0.1, \xi=0.3$ and $\xi=0.5$. 
Table 14: Effect of increasing social influence on long-run efforts with different values of speed of updating $\xi$.

\begin{tabular}{lccc}
\hline \hline$\chi$ & 0 and 0.3 & 0.3 and 0.6 & 0.6 and 0.9 \\
\hline$\xi=0.1$ & 0.0002 & 0.0001 & 0.0006 \\
$\xi=0.3$ & 0.0066 & 0.0002 & 0.0076 \\
$\xi=0.5$ & 1 & 0.0029 & 0.0355 \\
$\xi=0.6$ & 1 & 0.012 & 0.0033 \\
\hline \hline
\end{tabular}

The table shows the effect of increasing social influence on the long-run efforts in the baseline model $(\kappa=0)$. We compare each pair of long-run effort distributions for scenarios with trust parameter values $\chi$ equal to 0 and $0.3,0.3$ and $0.6,0.6$ and 0.9 when the speed of belief updating $\xi$ is 0.1 , $0.3,0.5,0.6$.

Table 15: Cross-effect of increasing speed of belief updating.

\begin{tabular}{lcc}
\hline \hline & 0.1 and 0.3 & 0.3 and 0.5 \\
\hline$\chi=0$ & 0.0458 & 0.0144 \\
$\chi=0.3$ & 0.0004 & 0.0048 \\
$\chi=0.6$ & 0.0001 & 0.0012 \\
$\chi=0.9$ & 0.0001 & 0.0004 \\
\hline \hline
\end{tabular}

The table shows the "cross-effect" of increasing the speed of belief updating $\xi$ for different levels of social influence parameter $\chi$. 


\section{References}

Acemoglu, D. and A. Ozdaglar (2011): "Spread of (mis)information in social networks," Dynamic Games and Applications, 1, 3-49.

Acemoglu, D., A. Ozdaglar, and A. ParandehGheibi (2010): "Opinion Dynamics and Learning in Social Networks," Games and Economic Behavior, 70, 194-227.

Anderson, S. P., J. K. Goeree, And C. A. Holt (2001): "MinimumEffort Coordination Games: Stochastic Potential and Logit Equilibrium," Games and Economic Behavior, 34, 177-199.

Arifovic, J.and Dawid, H., C. Deissenberg, and O. Kostyshyna (2010): "Learning Benevolent Leadership in a Heterogenous Agents Economy," Journal of Economic Dynamics and Control, 34, 1768-1790.

Blume, A. And A. Ortmann (2007): "The effects of costless pre-play communication: Experimental evidence from games with Pareto-ranked equilibria," Journal of Economic Theory, 132, 274-290.

Borgatti, S. P. And M. G. Everett (1999): "Models of core/periphery structures," Social Networks, 21, 375-395.

Bryant, J. (1983): "A Simple Rational Expectations Keynes-Type Model," The Quarterly Journal of Economics, 98, 525-528.

Burnside, C., M. Eichenbaum, and S. Rebelo (2016): "Understanding Booms and Busts in Housing Markets," Journal of Political Economy, 124, $1088-1147$.

Chen, R. And Y. Chen (2011): "The Potential of Social Identity for Equilibrium Selection," American Economic Review, 101, 2562-2589. 
DeGroot, M. H. (1974): "Reaching a consensus," Journal of the American Statistical Association, 69, 118-121.

Demarzo, P. M., D. Vayanos, and J. Zwiebel (2003): "Persuasion Bias, Social Influence and Unidimensional Opinions," The Quarterly Journal of Economics, 118, 909-968.

Golub, B. And M. JACKSOn (2010): "Naïve Learning in Social Networks and the Wisdom of Crowds," American Economic Journal: Microeconomics, 2, 112-149.

Golub, B. And M. O. JaCKsOn (2009): "How homophily affects learning and diffusion in networks," Tech. rep.

Grimm, V. And F. Mengel (2019): "Experiments on Belief Formation in Networks," forthcoming in Journal of the European Economic Association.

Huyck, J. V. And D. O. Stahl (2018a): "Conditional behavior and learning in similar stag hunt games," Experimental Economics, 21, 513-526.

- (2018b): "Conditional behavior and learning in similar stag hunt games," Experimental Economics, 21, 513-526.

Kriss, P. H., A. Blume, And R. A. Weber (2016): "Coordination with decentralized costly communication," Journal of Economic Behavior and Organization, 130, 225-241.

Riedel, A., I. T. Rohde, And M. Strobl (2016): "Efficient Coordination in Weakest-Link Games," Review of Economic Studies, 83, 737-767.

Rotemberg, J. J. (2017): "Group Learning, Wage Dispersion and Nonstationary Offers," Economica, 84, 365-392. 
Van Huyck, J. B., R. C. Battalio, and R. O. Beil (1990): "Tacit coordination games, strategic uncertainty, and coordination failure," The American Economic Review, 80, 234-248. 